This item was submitted to Loughborough's Research Repository by the author.

Items in Figshare are protected by copyright, with all rights reserved, unless otherwise indicated.

\title{
Development of a user-friendly, low-cost home energy monitoring and recording system
}

\section{PLEASE CITE THE PUBLISHED VERSION}

http://dx.doi.org/10.1016/j.energy.2016.05.027

\section{PUBLISHER}

(C) Elsevier

VERSION

AM (Accepted Manuscript)

\section{PUBLISHER STATEMENT}

This work is made available according to the conditions of the Creative Commons Attribution-NonCommercialNoDerivatives 4.0 International (CC BY-NC-ND 4.0) licence. Full details of this licence are available at: https://creativecommons.org/licenses/by-nc-nd/4.0/

\section{LICENCE}

CC BY-NC-ND 4.0

\section{REPOSITORY RECORD}

Fletcher, James, and W. Malalasekera. 2019. "Development of a User-friendly, Low-cost Home Energy Monitoring and Recording System". figshare. https://hdl.handle.net/2134/21434. 


\title{
Development of a user-friendly, low-cost home energy monitoring and
} recording system

\author{
James Fletcher and Weeratunge Malalasekera* \\ Wolfson School of Mechanical and Manufacturing Engineering \\ Loughborough University, Loughborough, Leics LE11 3TU \\ UK
}

* Corresponding author email: W. Malalasekera@lboro.ac.uk 


\begin{abstract}
This paper reports research undertaken to develop a user-friendly home energy monitoring system which is capable of collecting, processing and displaying detailed usage data. The system allows users to monitor power usage and switch their electronic appliances remotely, using any web enabled device, including computers, phones and tablets. The system aims to raise awareness of consumer energy use by gathering data about usage habits, and displaying this information to support consumers when selecting energy tariffs or new appliances.

To achieve these aims, bespoke electrical hardware, or 'nodes', have been designed and built to monitor power usage, switch devices on and off, and communicate via a Wi-Fi connection, with bespoke software, the 'server'. The server hosts a webpage which allows users to see a real-time overview of how power is being used in the home as well as allowing scheduled tasks and triggered tasks (which respond to events) to be programmed. The system takes advantage of well standardised networking specifications, such as Wi-Fi and TCP, allowing access from within the home, or remotely through the internet. The server runs under Debian Linux on a Raspberry Pi computer and is written in Python, HTML and JavaScript. The server includes advanced functionality, such as device recognition which allows users to individually monitor several devices that share a single node. The openPicus Flyport is used to provide Wi-Fi connectivity and programmable logic control to nodes. The Flyport is programmed with code compiled from $\mathrm{C}$.
\end{abstract}




\section{Introduction}

The management of electrical energy supply to meet the growing demand without contributing more to the green-house gas problem is a major task. To reduce green-house gases, more and more renewable sources of electrical energy are being added to networks. However, these new sources of supplies can be intermittent and variable. For example, analysis of the variability of wind power and how it could be integrated into electrical markets is detailed in [1]. This report discusses the main issues in terms of grid management and technological options required to address the variability of wind and other renewable sources and highlight the importance of developing technical solutions concomitant to the growth of intermittent renewables to ensure electricity system stability. Bass et al [2], using measured data from a gas turbine power generation plant in the UK, have shown how intermittent and variable sources of energy could impact on traditional power generation. Future management of electricity supply networks thus requires energy storage systems, variable tariff systems and cooperation from electricity consumers to limit low-priority power usage in times of high demand on the grid. In this context smart grid technologies are likely to play a major role. An electricity system, that is efficient, reliable, resilient and responsive, is a smarter grid [3]. Such a system would include an advanced metering system with the ability to use electricity more efficiently [3]. Electricity smart grids can be part of an overall smart energy system that include flexible generation systems and fluctuating renewable sources [4]. Home energy management is an important part of the solution to reduce greenhouse gases and incorporation of renewable sources of energy. For the success of the smart grid, home energy management and modelling systems with data recording facilities are also required. A number of recent studies have considered systems, software and hardware for home energy management. In the context of smart grids, a day-ahead real time pricing (DA-RTP) model based on smart meter data has been discussed in [5] and the study shows that a flatter demand curve and lower losses can be achieved by using such a system. The study by Almos et al [6] provides an analysis to illustrate the change of domestic load based on smart meter energy actions and the engagement of consumers is discussed in [6]. The development of an energy management system that incorporates consumer owned smart meters and distributor owned smart meters is described in [7] where applicable hardware and control systems have been demonstrated in a smart grid context. An approach that could be used to automatically analyse smart meter data to identify consumer characteristics has been presented in [8] where house hold characteristics could be used by suppliers to target energy efficiency campaigns. Depuru et al [9] have discussed in detail various features 
and technologies that can be integrated with a smart meters with the emphasis on security issues required in smart meter communication networks. Vega et al [10] have reviewed the most relevant literature and summarised infrastructure, communication media - protocols and variables managed by a system. Their study also analysed a large range of energy management models associated hardware and software and emphasised the need to incorporate monitoring, control and supervision of home appliances as well as the need to incorporate communication protocols to ensure the reliability of real time information collection through modern sampling tools and advanced algorithms. In other studies published in the literature, smart meter techniques have been discussed in details. For example, various features and technologies that can be integrated with a smart meters has been discussed by Depuru et al [9]. Vessileva et al [11] have discussed the need the need for providing energy consumers with required information about their energy usage and studied the consumer categories and how it could be used to develop effective demand response measures. Further relevant studies include the work of Beckel [8], Pereira et al [7] and Zhao et al [12]. At a higher level (supplier or distributer), the smart grid concepts appear be a good solution for integrating variable and distributed energy sources. An overview of the emerging smart grid and the potential for the smart grid to act as an enabling technology for renewable energy integration, price-responsive electricity demand, electrified transportation and distributed energy production is discussed in [13]. User mode distributed energy management via smart grids [14] and potential use of information and communication technologies (ICT) with regards to smart grids are discussed in [15]. At a domestic and consumer level smart meters have emerged as the technological solution providing necessary data for the incorporation of renewable energy systems, monitoring and control of metering technologies and demand management (Batista [16]). Availability of reliable, low cost flexible hardware and software solutions capable of metering, data collection, data analysis, storing and transmission are required for the success of the smart grid. Kim et al [17] have discussed the overall structure of a smart grid and the role of Universal Plug and Play (UPnP) devices for Home Energy Management Systems (HEMS). Most other studies relevant to the present study have used IEEE 801.15.4 and ZigBee technologies for device control and energy management (Han and Lim [18], Batista et al [16]). Jang and Healy [19] in their studies have discussed in detail the challenges and obstacles in the implementation of wireless sensor networks in buildings. The main challenges are building structure, reliability degradation, security, battery life time, initial cost and ease of use. Some energy management systems have explored the use of Power Line Communication (PLC) technologies for data collection and recording in 
home energy management. These include the work of Han et al [20], Papagiannis et al [21], Al-Mulla and EISherbini [22]. Further details are available in the review of powerline technologies for smart grid applications by Yigit et al [23].

At consumer level various forms of smart meters have been introduced by electricity suppliers to inform customers of their energy usage profiles and these have enabled the suppliers to obtain useful demand profiles. However some smart meters do not provide a breakdown of which appliances contribute to the overall demand profile. This has been a problem and smart meters are already termed to be 'not so smart meters' [24]. There is great interest in developing useful, secure, low-cost technologies to monitor and record domestic energy usage and allow users to have more control over their energy usage. Perhaps in the future such systems may even be able to automatically switch energy provider on their owners behalf, depending on which companies offer the cheapest, greenest energy. The technology could also be used to deliver targeted advertising to users supported by savings estimates based on the real usage data that the system collects.

None of the existing 'smart meter' solutions offered by energy suppliers to private homes in the UK are able to control devices around the home. Input into the National Grid in the UK is almost entirely comprised of plants which are unable to quickly change their rate of supply, (for example, gas, coal, nuclear) or worse, have an unreliable rate of supply e.g. wind. Since mass produced electricity is not easily stored, the grid must attempt to balance supply with demand by forecasting demand. Demand trends typically follow the day/night, weekday/weekend and summer/winter cycles, but are also affected by social events, such as breaks during soap operas and sport events. If the grid could temporarily enable and disable high-power, non-time critical devices around the home, such as water heaters and air conditioners then they would be more effectively able to balance supply with demand, whilst avoiding resorting to inefficient energy storage or 'brown-outs'.

Appliance monitoring and control systems have the potential to reduce costs, energy consumption and carbon emissions [25]. They can achieve this by providing sophisticated control functionality, and by collecting useful data that can be used to inform appliance purchase decisions and select more economical energy providers. Energy providers can use the data to provide low cost tariffs and in an ideally open market-place for electricity-supply, users should be allowed to switch suppliers at a short-notice to take advantage of low cost tariffs and change their usage profile to minimise their energy costs. 
As mentioned above most currently available 'smart meters' are able to collect data but consumers could not identify elements of their energy usage or analyse data to make useful interventions to change their behaviour. In many cases smart meters show a cost at a particular time but do not provide information on which appliances are responsible for the cost or collect item specific data and present them in a useful manner to understand their energy profiles. There is a requirement to develop low-cost smart meters which can collect detailed data, communicate with simple devices such as mobile phones, tablets and computers at home and consumers should be able to see energy usage, broken down by appliances and time of use so that they can make changes in their behaviour to use energy more efficiently or buy new and energy efficient appliances to save energy on longterm. This project aims to develop a low cost secure hardware and software solution, making as much use of existing hardware as possible, which can be used to monitor and control mains electrical devices, at the same time collecting information about the way energy is used in the home. The information collected aims to be of sufficient quality to help users make informed decisions about potential appliance purchases and energy tariffs based on the user's actual usage habits.

\section{The Methodology and System Design}

\subsection{Structure of the system}

The main aim of this research is to develop a system that is capable of identifying where energy is used around the home, as well as storing and presenting the data collected in an accessible and visual way using webpages which can be accessed remotely from phones and computers. The system also aims to allow users to switch devices around the home on and off through the web interface provided. The system consists of a single server and one or more nodes which monitor and control one or more attached devices. The overall system and its operation is shown in Figure 1.

Figure 1. An illustration of the overall system concept

The system consists of a single server which is in communication with multiple nodes and multiple clients. In the context of this research, a device is any mains electrical appliance 
that can be controlled by this system, for example desk lamps, white goods, fans or televisions etc.

A node is the hardware which physically switches the mains power to attached devices at the request of the server. The node also monitors the power usage of the device and sends this information to the server. A home can have many nodes in multiple formats, for example a unit that sits between the wall socket and device plug, circuitry concealed behind a wall switch or circuitry physically built into an appliance.

The server runs permanently in order to receive information from nodes and issue commands to nodes. The server also hosts a web interface capable of serving clients, manages scheduled tasks and events, and records power usage and user activity by submitting records to a database.

A client allows users to issue commands to the system such as turning devices on or off. Clients can also query usage data and configure rules. Since the server hosts a web interface, clients can take the form of any device capable of connecting to a wired or wireless network and displaying HTTP or HTTPS webpages. Serving clients via a web page as opposed to an application (app) releases any dependency of the system on a particular client operating system, such as Google Android, Apple iOS or Microsoft Windows. These operating systems would all require dedicated versions (using different languages) of any bespoke client software developed for this project, but all support web content by default. Using the display and connectivity features of common-place devices (such as laptops and smartphones etc) eliminates the need to build custom client hardware at additional project and end user cost. A detailed system layout which depects above features is shown in Figure 2.

Figure 2 Detailed system layout

\subsection{Device Recognition Theory}

Often in the home, multiple devices share a single plug socket via a multi-socket extension. In such scenarios each device would require an individual node to monitor the power usage. It is theoretically possible to identify and monitor multiple devices attached to a single node by analysing and remembering the way devices use power. 
This is permissible because the electrical hardware within devices around the home is made up from different combinations of resistive, inductive or capacitive components. These components can draw current at a different phase to an input Alternating Current (AC) voltage. Those that do not follow the input voltage (resistive) either lag (inductive) or lead (capacitive). When their current draw waveforms are combined it yields a unique overall current draw waveform for that device.

Figure 3 shows the current waveform from a fan-cooled overhead projector next to that of an assortment of audio/visual equipment including a video tape player, an amplifier, desktop computer and a monitor.

Figure 3. Measured current transducer waveform for two devices

Despite the fact that both devices are supplied with the same 230 VAC waveform, their current draw is significantly different. The overhead projector is the simplest device, and with no sophisticated control electronics, only appears to show two distant frequency components. The light in the projector is a resistive load (in phase with the mains at 50 $\mathrm{Hz}$ ), whereas the fan is an inductive load (lagging behind the mains). The audio visual (AV) equipment however, contains many hundreds of resistors, inductors and capacitors and therefore displays a much more erratic waveform, very different from the sinusoidal AC voltage supplied.

To the human eye, these waveforms have an appearance which makes them easily distinguishable. Computer software can differentiate between the two using Fast Fourier Transform (FFT) algorithms [26] FFT is used in this work to identify key frequencies, uniquely characteristic of specific devices.

By performing device recognition, the server software can extract more information about the way power is used around the home, delivering a better user experience. Device recognition can work in a variety of configurations. For example, multiple devices plugged into a single node. In this configuration the number of physical nodes required to monitor devices individually is reduced. Events relating to individual devices can be detected irrespective of what adjacent devices are doing. A user may apply this feature to calculate which device, on a chain of many devices, is using the most power without having to purchase and run separate nodes or manually swap the connected device. 
Theoretically, this configuration could be scaled up to monitor all of the devices in a household, from a single node. The node would monitor AC mains current from a clip-on hall effect sensor attached to the mains supply to the household.

Another possible application for device recognition is for predicting failure. In this configuration a single device would be plugged into one node and the server would continually monitor the device to ensure its profile matches a known working profile for that device. Any deviation from the known profile may indicate a physical deterioration of the device - possibly a precursor to failure.

\section{Node Hardware}

As mentioned earlier in this work, relatively low cost readily available hardware is explored to provide necessary functionality and requirements of the above explained system. In this work the node hardware consists of three major components: logic (including communication), is provided by the openPicus Flyport; switching is provided by a Sharp Solid State Relay; and current measurement is carried out by an HX 03-P/SP2 current transducer. These components have been selected for their low cost, availability and suitability for interfacing between mains current and low voltage, DC control circuitry.

\subsection{Logic: OpenPicus Flyport Wi-Fi}

The Flyport Wi-Fi [27] used is equipped with 18 digital input/outputs which are capable of Pulse Width Modulation (PWM). The Flyport also offers four 10-bit analogue inputs. A 10bit analogue to digital converter $(A D C)$ can resolve $2^{10}$ or 1024 divisions which is a relatively poor resolution. The Flyport has an SPI interface which can be used to interact with an external 24-bit ADC giving significantly better resolution. However, as discussed later in section 3.5 , a technique exists which can greatly improve the sensitivity of a 10-bit ADC.

\subsection{Load Switching: Sharp S202S01F Solid State Relay}

Hear a SHARP S202S01F solid state relay [28] used to switch the high voltage current. A solid state relay is used as it does require as much voltage or current as a mechanical relay to hold 'on'. This is important, because the relay is driven by the Flyport which has a digital I/O voltage of $3.3 \mathrm{~V}$ and a maximum available current of $18 \mathrm{~mA}$ [29]. The S202S01F can switch loads of up to $8 \mathrm{~A}$ and has an isolation voltage of $4.0 \mathrm{kV}$. The device is 
therefore ideally suited for switching AC mains devices. Unfortunately SSRs tend to fail in the ON state which is a potential hazard for use in the home.

\subsection{Current Measurement: HX 03-P/SP2 Current Transducer}

The LEM HX 03-P/SP2 current transducer [30]. The transducer is effectively an analogue Hall Effect sensor which outputs a voltage proportional to the current in the mains circuit. The current transducer has a range of $\pm 9 \mathrm{~A}$ which corresponds linearly to a DC output voltage of $2.5 \pm 0.625 \mathrm{~V}$, where $2.5 \mathrm{~V}$ corresponds to a measured current of $0 \mathrm{~A}$.

In addition to these three major components, an LM358N Integrated Circuit [31] is used to shift and amplify (or attenuate) the current transducer signal into the range that the Flyport can register.

\subsection{0-bit ADC Resolution for Measuring Power}

The Flyport (selected to provide the logic and measurement capabilities) only contains a 10-bit analogue to digital converter (ADC). A 10-bit ADC can only resolve 1024 divisions. In any $A D C$, readings will lock to the nearest integer division. For a design load of $1 \mathrm{~kW}$ (where $80 \%$ of the Flyport sensitivity represents $1 \mathrm{~kW}$ ) one division is equal to $13.8 \mathrm{~W}$. Therefore the error is $\pm 6.9 \mathrm{~W}$. Such a large error is unacceptable in a system that will be used to measure the power consumption of devices, such as low energy light bulbs, which may use less than $10 \mathrm{~W}$ in total.

One technique to improve the sensitivity of power measurements is to exploit the fluctuating nature of the current transducer output wave, by taking multiple measurements throughout a cycle and calculating the RMS current. This approach works because on average, between divisions, rounding-up errors will be largely offset by rounding down errors.

To illustrate this, a synthesised current draw waveform representing a 100 watt tungsten light bulb powered by 230 VAC was defined in Microsoft Excel. The waveform consisted of 350 samples equally spaced over an $80 \mathrm{~ms}$ period. The magnitude of each sample was rounded to the nearest integer as would be the case in the Flyport's ADC. The amplitude of the wave was such that a $1 \mathrm{~kW}$ variant would occupy $80 \%$ of the Flyports sensitivity, peak-to-peak. The calculated error as a function of ADC resolution is given for the multiple point technique and a single point of measurement as shown in Figure 4. 
Figure 4 - Error as a function of ADC resolution

As the figure illustrates, by using multiple sample points, a very high level of sensitivity can be achieved with a reasonably low resolution ADC. By using multiple points, a 10-bit ADC with 1024 divisions has the equivalent sensitivity of a 16-bit ADC with 65536 divisions. For a 10 -bit ADC, the error is $0.11 \%$.

\section{Server Hardware}

The primary function of the server is to coordinate communication between nodes and clients. The server also stores and queries data as well as manages scheduled tasks and events. In recent years, various low cost computer hardware capable of running basic Linux operating systems have appeared in the market. In this research we have explored such devices. The Raspberry Pi [32], released in February 2012 is used as the server hardware within this project. The Raspberry $\mathrm{Pi}$ is a credit card-sized, silent, low energy and inexpensive PC powered by a Broadcom BCM2835 SoC (System on Chip) which contains $512 \mathrm{MB}$ of SDRAM as well as a $700 \mathrm{MHz}$ ARM1176JZF processor [33]. The Raspberry Pi is designed to run variations of Linux. For this project, the Raspberry Pi is configured to run Wheezy Raspbian [34] (build date 9/2/13) which is a free variant of Debian Linux licenced under the GNU General Public License, version 2. This version requires minimal configuration and has USB, network and Python support built in.

\section{Node Hardware}

Two prototypes were developed for the project. Figure 5 shows the final prototype.

Figure 5 Final prototype with cover removed

Since the node is powered by AC mains voltage, the shape of the waveform output from the current transducer is expected to cycle (repeat) at a rate of $50 \mathrm{~Hz}$. In order to collect data at sufficient resolution for analysis the Flyport should aim to collect approximately 100 data points per cycle. To capture 100 points per cycle requires a sample rate of $5000 \mathrm{~Hz}$ sustained for at least 20 milliseconds (ideally $80 \mathrm{~ms}$ ). 
The data collected is used by the system for two functions: firstly, to calculate the power usage of the attached devices; and secondly, to identify attached devices. In this prototype a rectifier board (which converts the area enclosed by the sinusoidal current waveform to a flat $\mathrm{DC}$ voltage) had been added so that in the event that the Flyport was unable to sample data at a sufficient rate, power could still be measured. However, resorting to this method would make device recognition impossible. Fortunately the Flyport sample rate was more than sufficient and therefore the rectifier board was not required.

\subsection{Amplifier Tuning}

The Flyport sensitivity is limited to 1024 divisions, therefore it is important to use as much of the range as possible to increase the resolution of the signal. Tuning also helps to ensure that the signal does not go beyond the Flyport's tolerable parameters i.e. an input voltage between $0 \mathrm{~V}$ and $2.048 \mathrm{~V}$, so to prevent damage to the internal ADC. Tuning is achieved using an amplifier circuit capable of applying a fixed gain and offset to the current transducer signal.

Figure 6 shows a simulated output directly from the current transducer when measuring a 60 watt tungsten light bulb. As in this example and the next, a tungsten bulb is used as it has an almost entirely resistive load, and therefore, the current transducer output can be expected to fluctuate perfectly sinusoidally. In the figure, the red lines are the DC cut-off voltages for the Flyport and the green wave is the DC output from the current transducer.

Figure 6 - Current transducer output

As the Figure 10 shows, the peak to peak voltage from the transducer is very small (approximately $0.03 \mathrm{~V}$ ). This peak to peak range spans fewer than 18 of the 1024 divisions available giving very poor resolution. However, the main problem with this signal is that it is outside of the Flyport's range. The Flyport ADC will output 1024 for every point it captures and could also be damaged from being subjected to 'over-volting'.

Figure 7 shows the same signal after applying an appropriate gain and offset. In this signal the peak to peak height spans 824 of the 1024 divisions, and fits within the Flyport's 
tolerable range, with an added margin of safety for noise and drift (due to thermal effects on components and other factors).

Figure 7 - Amplifier output (to Flyport)

This is implemented in the node prototype using the offset and gain potentiometers as shown in in Figure 8.

Figure 8 Node amplifier board showing important potentiometers

\subsection{Provisional Calibration}

To measure power, the server requires that the Flyport samples a waveform from the current transducer using its internal ADC. For any one measurement, the ADC returns an integer value between 0 and 1024, inclusive. The ADC value is linearly proportional to the output from the amplifier, in turn linearly proportional to the instantaneous current draw of the attached device. The server calculates an RMS value for the waveform, measured in divisions. To convert this value to amps within the server requires calibration parameters. The relationship between the RMS value, $x$ (divisions) and the current draw of the attached device (amps) can be found using eqn, (1).

$$
I_{R M S}(\text { Amps })=m x_{R M S}(\text { Divisions })+c
$$

To solve for both $m$ and $c$ the node must be tested under two different loads. Firstly, with no device connected; and again with a device which draws a stable load. In each of these states, the actual current draw was measured using a Prodigit 2000MU power monitor. The accuracy of the $2000 \mathrm{MU}$ when measuring RMS current is claimed to be within $1 \%$ and typically around $0.3 \%$ (within the operating temperature and humidity) [35]. The test device used during calibration of the system was a $60 \mathrm{~W}$ tungsten light bulb.

The calibration factors $m$ and $c$ were found to be $2.541 \times 10^{-3}$ and $-1.108 \times 10^{-2}$ respectively. These values are stored in the server database. 


\section{Node Firmware}

The Flyport is controlled by firmware that runs from its internal non-volatile memory. The firmware is developed in the openPicus IDE and flashed to the Flyport via a USB programmer. The Flyport has $256 \mathrm{~KB}$ of internal non-volatile memory of which a very small amount (approximately $1 \mathrm{~KB}$ ) is occupied by the device's boot loader.

The Flyport firmware performs the following functions;

- Establish and maintain a permanent contact with the server

- Periodically send the Flyport's Media Access Control (MAC) address to the server, initially for device recognition and then as a keep alive signal.

- Respond to a current trace request from the server by replying with a burst of 350 samples captured from the current transducer over $80 \mathrm{~ms}$

- Switch the solid state relay on or off at the request of the server

\section{Server Software}

In this work the server software is programmed using Python. Python is an open source high level programming language which is almost entirely platform independent. Python supports the essential advanced programming functionality required for this project such as managing TCP sockets, serving web clients, advanced mathematics and multithreading.

The server source code was written using Microsoft Visual Studio Express and the Python Tools for Visual Studio (PTVS) add-on which adds syntax highlighting and other features to Visual Studio for Python. The expansion is available as a free download from CodePlex, Microsoft's free code project hosting website [36].

\subsection{Configuring the Server Environment}

Wheezy Raspbian (build 9/2/13), based on Debian Linux (RaspberryPi) is used as the operating system on the Raspberry Pi. Raspbian supports Python by default and is supplied with minimal features, thus reducing unnecessary power consumption.

The operating system is flashed to a Secure Digital (SD) card and inserted directly into the Raspberry $\mathrm{Pi}$. The following significant changes were made from the default Raspbrian configuration in order to allow the server software to run; 
- IP address fixed to ensure it is always reachable on the network

- MySQL server installed (to store data from the server software)

- Python MySQL [37] libraries installed to allow Python to talk to the MySQL database engine.

- Numpy installed to add some scientific mathematical funcitions to Python [38].

- SciPi installed to add advanced mathematical functions to Python including FFT [39].

- Samba installed to allow Microsoft Windows based systems to modify files on the Raspberry Pi via a Windows network [40].

\subsection{Data Storage and Retrieval}

Data and settings are stored in a database provided by the free MySQL database engine. The database engine is separate from the server but runs beside it on the Raspberry Pi. At start up, the server connects to the database to read and manipulate records using Structured Query Language (SQL).

The database schema and other settings are configured remotely via the freely available MySQL Workbench software.

Using a database engine to store data as opposed to storing data in a proprietary format allows third party software to interface with, analyse and manipulate the data. This approach has also helped to implement advanced data storage and retrieval functionality within the server software using available software tools.

\subsection{Web Interface}

The web interface is the primary way in which users interact with the system. Though the web interface, users can switch nodes on or off, monitor power usage, set up scheduled tasks and events and train the system to recognise devices.

The major advantage of using a web based interface to interact with the system is that it does not require bespoke software to be written for specific client operating systems. Instead all web enabled devices including PCs, Macs, phones and tablet computers can access the system without installation of any additional software, since all of these devices come with web support.

The web interface is served using a proprietary webserver written in Python and integrated into the server software. This proprietary webserver is based on the standard Python 
SimpleHTTPServer module which handles incoming HTTP requests. This allows greater control over content delivery than would be permissible trying to link the server software to a third party webserver package (such as Apache).

The website's visual interface is coded in HTML and Cascading Style sheets (CSS) [41] using Notepad++ [42]. The HTML \& CSS files are stored in the same directory as the server software allowing for easy customisation. When the web interface is requested, the server software retrieves the relevant files and hands them to a SimpleHTTPServer object which streams them to the client via the HTTP protocol. Live communication between the client and server is achieved using Asynchronous JavaScript and XML (AJAX). Once the user's browser software receives the page in full, an AJAX object is created which connects back to the server periodically and requests updates such as the current system power usage. This technology allows the user to sit back and monitor the state of the system without having to continuously refresh the page.

\section{Demonstration of the Software and Associated Functionality}

\subsection{The Web Interface}

Figure 9 shows the overview screen of the developed web interface. The screen lists basic information about the system as a whole, for example the number of nodes that are currently connected, the overall system power usage, the $\mathrm{CO}_{2}$ output etc.

Figure 9 - Overview page

Also in the summary table, the overview page also lists all of the nodes that are connected. Each entry in the table has a dedicated power control and power usage reading. Nodes not currently connected are hidden to keep the user interface clean and easy to navigate.

The overview screen also displays power usage across a time period in graph format as shown in Figure 10. The graphical representation aims to help users associate periods of high consumption with particular activities or events.

Figure 10 - Power usage graph 
Graphing is provided using the Highcharts 3.0.1 [43] and Javascript API. Highcharts can be used to display data in real-time and is compatible with all popular desktop and mobile browsers.

Users can select between a variety of time periods from the menu shown in Figure 11.

Figure 11 - Time period menu

The time period list box governs the date ranges used to calculate energy use, cost and $\mathrm{CO}_{2}$ output, as well as the date range used on the graph shown previously.

Data on the overview screen are updated automatically without requiring the user to refresh the page or click any buttons. This is achieved using Asynchronous JavaScript and XML (AJAX).

The settings page, shown in Figure 12, is accessed from the menu to the left.

Figure 12 - Settings page

The settings page allows users to set up and configure nodes, add locations, set up device recognition, test device recognition and modify variables such as energy costs and grams of $\mathrm{CO}_{2}$ per kWh. By default the cost of electricity in terms of money and $\mathrm{CO}_{2}$ is $13.9 \mathrm{p}$ per $\mathrm{kWh}$ and $0.55 \mathrm{~g} \mathrm{CO}_{2}$ per kWh respectively [44,45]. Like the overview page, this page communicates with the server using AJAX.

\subsection{Mobile Client Interface}

Figure 13 shows the same data accessed via a mobile phone. The server automatically detects mobile clients and serves an alternative touchscreen-optimised version of the overview and settings pages with larger fonts and buttons as shown in Figure 13.

Figure 13 - Mobile version of the overview page

\subsection{Security}

Research for this work has shown that consumers are concerned about the security of similar internet enabled systems, which have the capability to control and / or monitor electronic devices within the home. Providing the option to view the web interface via 
HTTPS instead of HTTP is one step towards ensuring security. The HTTPS protocol encrypts communication between the server and client making it impossible for messages to be read or modified in transit by third parties.

When the web interface is loaded on a browser such as Chrome, the user is shown that the page is secured with HTTPS. Chrome indicates this by adding the ${ }^{8}$ https:// prefix at the start of the server address as shown in Figure 14.

Figure 14 - HTTPS connection information in Google Chrome

This figure also shows details about the encryption level. In this case 256-bit encryption is used with a self-signed certificate.

\subsection{Node - Server Communication}

Messages between individual nodes and the server are sent via a TCP (Transmission Control Protocol) socket as opposed to a UDP (User Datagram Protocol) socket. Although TCP has a slower response time, it guarantees that information arrives well-formed and in the same order in which it was sent.

With UDP, information is sent as fast as possible. This can lead to more recently sent packets arriving before older packets depending on their route through the network. With UDP it is impossible to know if information has been lost in transmission. TCP response time and throughput is still exceptionally good compared to UDP [46] and more than sufficient for this project.

The node TCP socket is opened in an individual thread in order to concurrent connections and implement full asynchronous communication support between the node and server.

Before a node can be used by the system it is first 'identified'. Identification matches a node to its associated settings from the database such as name, location and calibration data. Nodes are identified based on their Media Access Control (MAC) address. MAC addresses are used for identification because they are guaranteed to be unique. This allows nodes to be identified even if their IP addresses change (for example on a DHCP network) or even when connecting from an external network via the internet.

Figure 15 shows how a user request to turn a device on enters the system and is communicated to the node. 
Figure 15 - Power switch input / output path

\subsection{Signal Processing, FFT and Power Calculation}

Raw data arrives at the server from the nodes as a series of 10-bit ADC divisions vs time offset in milliseconds. The server then subjects to signal to the following processing steps.

- Time-zero all points (the first point is not naturally stamped with time $=0$ as there is a small delay between the Flyport recording the start time and sampling the first point).

- Artificially resample the signal at constant intervals over $2^{12}$ (4096) points by interpolating between data points. This step results in some minor loss of quality, but is essential for the FFT analysis.

- Calculate the RMS current in 10-bit ADC divisions using eqn. (2) where $N$ is the total number of data points and $I$ is the current at a given data point, $x$.

$$
I_{R M S}(\text { divisions })=\sqrt{\frac{1}{N} \sum_{x=1}^{N} I_{x}{ }^{2}}
$$

- Convert the RMS current in 10-bit ADC divisions to RMS current in amps using eqn.

(3) where $m$ and $c$ are calibration factors found using a reference device:

$$
\begin{aligned}
& I_{R M S}(\text { amps })=m I_{R M S}(\text { divisions })+c \\
& \text { Permissible because at } I \text { (amps })=0, I(\text { amps })=I(\text { divisions })
\end{aligned}
$$

- Calculate the Power in watts using equation 4. (AC mains RMS voltage is assumed to be $230 \mathrm{~V}$ ).

$$
\text { Power }(\text { watts })=I_{R M S}(\text { amps }) \times V_{R M S}(\text { volts })
$$

- Extract the frequency spectrum from the signal using the Scipy FFT function.

- Make a list of characteristic frequencies and magnitudes which have a magnitude above a predefined cut off. (This list is used for device recognition).

Figure 16 shows the frequency spectrum generated by a FFT analysis of the waveform of a low energy light bulb. In this graph, a relative magnitude is plotted 
against frequency. The horizontal red line marks the position of the cut off threshold.

Figure 16 - Frequency domain of test signal

The two vertical red lines represent the frequency and magnitude of each peak which rises above the cut off frequency.

\section{6 Device Recognition Implementation}

\subsubsection{Profiling}

For a device to be recognised, it must first have a 'profile'. Device profiles are a list of the key frequencies and magnitudes that were observed by a node when powering the device. Users instruct the server to profile a device using the settings page within the web interface. Figure 17 shows the final state of the device profiler tool after a device has been profiled.

\section{Figure 17 - Device profiling tool}

This interface allows the user to select the node that a device is attached to, to name the device and to view the frequencies and magnitudes that correspond to the device profile. The key frequencies are found as described previously in section 8.5 and are stored in the database.

\subsubsection{Device Recognition}

Device recognition follows the process as described by Figure 18 .

Figure 18 - Device recognition procedure

When device recognition is required, key frequencies and magnitudes are calculated from data received from a node. This information is passed to the IdentifiyDevices function internally.

IdentifyDevices then loads in all of the device profiles (potential devices) from the database and tries to eliminate as many devices as possible. This is critical for combination testing in the next step. A device profile can safely be eliminated if either of the following criteria are true. The potential device; 
- requires a frequency which is not present in the live data; or

- has a frequency which matches the live data, but at a magnitude which is far in excess of the magnitude for the same frequency in the live data.

Once devices have been eliminated, IdentifyDevices tries to start combination testing. Combination testing works by superimposing the signals of the potential devices in every possible combination and comparing combined frequencies and magnitudes to the live data. Table 1 shows the four possible states that two potential devices could be in.

\section{Table 1 - Device states}

For the software, it is easier to refer to these combinations as bit encoded strings. A 'string' is a series of characters and by extension, a bit encoded string is a series of binary characters. The position of the character in the string refers to the device and ' 1 ' and ' 0 ' represent 'on' and 'off' respectively. This is convenient for iteration as a bit encoded string is effectively a binary number - interchangeable with an integer value.

The software skips the first combination (integer value zero) as this is the equivalent of the software failing to recognise at least one device in the live data. If after iterating through all combinations without identifying a device, it is assumed that none of the potential devices are present and the function returns an empty list.

The number of possible power state combinations that a series of potential devices can be in is therefore $2^{N}-1$ where $N$ is the number of potential devices. As mentioned previously, it is therefore very important that as many potential devices as possible are eliminated, as each additional device doubles the number of possible combinations. More devices therefore exponentially increases the (theoretical maximum) time it takes to recognise devices.

Once the system knows what devices are plugged in, it is then possible to convert the key frequencies and magnitudes back into a waveform for each attached device. This can then be processed in the same way that the raw data is processed to find out how much power each device should be using. However since the key frequencies and magnitudes function ignores noise and small magnitudes, the power of the synthesised waves will be less than the actual total power. As a rough estimate, the system can increase each synthesised wave power proportionally until their sum gives the actual measured system power. This 
technique allows users to see how much each attached device is using, separately, with just one node.

\section{Evaluation of the complete system}

\subsection{Testing and evaluation of functionality and response}

The performance of the system was evaluated in laboratory conditions. Only one fully functional node prototype was constructed to prove that the Flyport could switch appliances on and off as well as monitor the power of attached devices. This system was tested using two devices, a low energy light bulb and tungsten light bulb connected to a power strip, in turn connected to the node. Performance of the system was good and behaved as expected with regards to both switching and monitoring. These devices were tested intermittently over three weeks and no appreciable drift in power consumption was detected (+/- 8 watts). Power consumption was verified using a Prodigit 2000MU power meter. Some testing was also undertaken using AV equipment and proved successful. During one experiment, the Solid State Relay failed in its 'on' state. This is a potentially dangerous failure mode, especially if such a system were to be used to control a heater. It is therefore recommend to always use a mechanical relay that requires power to hold 'on' in such applications. Due to the limited power of the Raspberry $\mathrm{Pi}$, it was found that an unacceptable lag of up to 3 seconds can exist between issuing a command on the web interface and seeing activation of an attached device. It was found that by disabling the power usage logging features of the system, this lag can be reduced such that it is imperceptible. The primary cause of lag was determined to be the time in which the Flyport takes to receive and act on an instruction to capture power data, followed by the time it takes to send this back to the server and for the server to store it in the MySQL database. During this time, no instructions can be acted upon. Several remedies are suggested including moving the database storage functionality to its own thread and having the Flyport check for further instruction every nth cycle of its power usage capturing loop.

An additional Flyport and a node simulator script were used in order to demonstrate that the system can handle multiple nodes and act on user specified rules. Two Flyport units were purchased for the project. The firmware developed for the Flyports makes no attempt to detect whether or not the Flyport is running from a node or an FTDI programming board. Therefore as long as the Flyport is powered, it will attempt to connect to the server and report its state and act on commands. The Flyport has two on-board LEDs, the first of which is programmed to illuminate when connected to the server and second when the 
attached device is meant to be activated. Using this feedback it was possible to test the control components of the system on two nodes, despite one node not having the associated monitoring and switching hardware.

In addition to this, a simple Python script was written to simulate the behaviour of nodes. The script was designed such that it connects to the server software and reacts to instructions from the server in the same way a physical node would, but in place of turning a device on or off, the code simply displays a message on the screen explaining what it has been instructed to do. Multiple instances of the node simulator script can be run simultaneously. By combining this infrastructure, it was possible to show that the server is issuing commands to nodes in response to user specified rules such as 'on timer events' and 'on state change' events.

\subsection{Evaluation of device recognition}

Below the device setup section on the settings page is the recognition test tool, which can be used to check device recognition. Figure 19 shows the device recognition test tool after querying the devices attached to 'Unnamed Node'.

Figure 19 - Device recognition test tool

To use the tool, the user is required to select a node to query and then click the 'check' button. If the device recognition function identifies any devices attached to the selected node, they appear as a list on the page.

Using this tool, the system was shown to successfully identify the Light and Low Energy Light devices in all four possible combinations. Device recognition applied to these two devices proved to be very repeatable and accurate.

Device recognition only works for devices which draw a static load. This may include for example light bulbs, fans and heaters. Devices with irregular duty cycles, such as laptop chargers, computers and washing machines are significantly harder to profile with this implementation of device recognition.

\subsection{Event Handling and Task Scheduling}

Event handling and task scheduling are essential elements of the 'Automation' component of this work. Event handling and scheduling are two similar concepts which are both ultimately used trigger a task. A task performs some kind of action, the available actions 
are; sending an email, switching the power state of a node, outputting a message to the server console, saving the overall system power usage to the database, and running Python code directly.

Task scheduling triggers tasks at periodic intervals, or at a specific date and time, either repetitively or just once.

An example of a user-driven scheduled task might be to turn a light on and then off again at specific times. For example, to make an unoccupied house appear occupied, or to turn decorative exterior lighting off after midnight.

\section{Conclusions}

This research project set out to develop a hardware and software solution to automate, monitor and control electrical/electronic devices around the home. The ultimate aim was to develop a lost cost, versatile system which could raise consumer awareness of energy consumption and also reduce energy consumption, by supporting consumer decisions such as the choice of energy supplier or appliance specification when purchasing new electrical equipment, based on real usage data.

Much of the base level development has been achieved. For example, the production of a working node prototype and writing server software which supports multiple node connections as well as serving a user interface via a web page, performing device recognition, calculating power usage, managing scheduled tasks and recording data has been achieved and successfully demonstrated. In the development of the system, low-cost components and open-source software have been used. The system developed is very detailed in terms of data gathering, storing and displaying to the user and could be improved further with additional features required by the home energy market.

However the system has several limitations. The node prototypes are relatively large and should be packaged in a more discrete enclosure. This being a research project no attention was paid to address this aspect and it could be easily addressed with specialist components. Whilst the vast majority of the core software functionality is in place, there is still plenty of room for improvement. For example, a richer user interface, better task configuration, recording macros and the introduction of a dedicated cost benefit calculator could be added. The calculator would be powered by data collected over the life of the system and could be linked to energy supplier websites and domestic appliance manufacturer websites. 
The major conclusions from this project are that;

- It is possible to monitor and control multiple devices around the home in real time from a web interface using the developed hardware software packages,

- the software developed for this project enables a detailed log of user activity and device power usage to be compiled, which can be used to determine consumption trends and inform purchase decisions, and,

- using Fast Fourier Transforms and signal analysis, it is possible to identify the types of devices plugged into nodes and how much power they use individually, based on the way they use power.

\section{Acknowledgements}

We acknowledge the support from Clker.com, http://www.clker.com/ for hosting free for commercial use images used in the website component of this project. Including the 'green earth' image that forms part of the HAMC logo. Highsoft Solutions AS, http://highsoft.com/ for making their JavaScript charting API free for use in projects such as this.

\section{Nomenclature}

Abbreviations

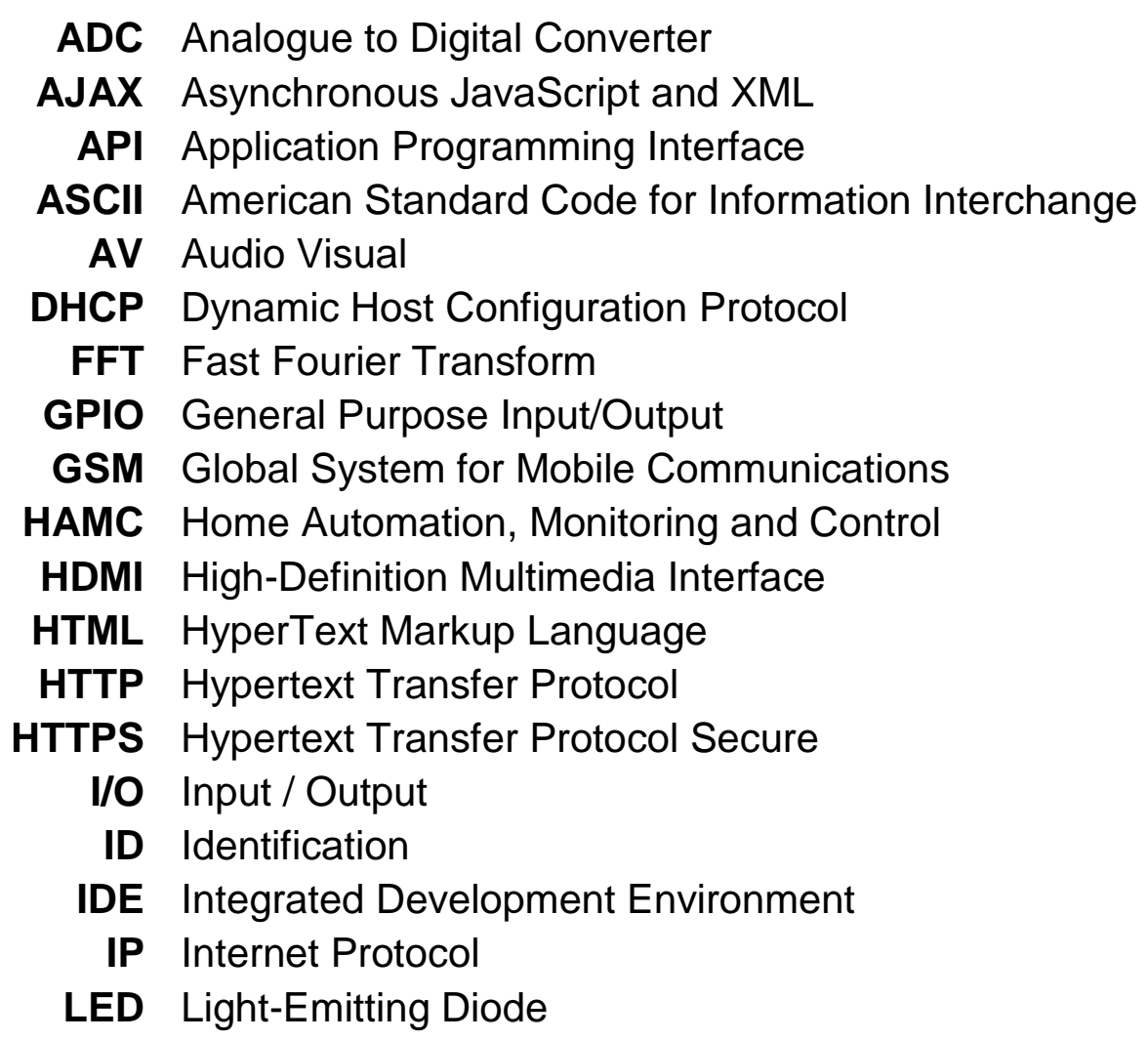




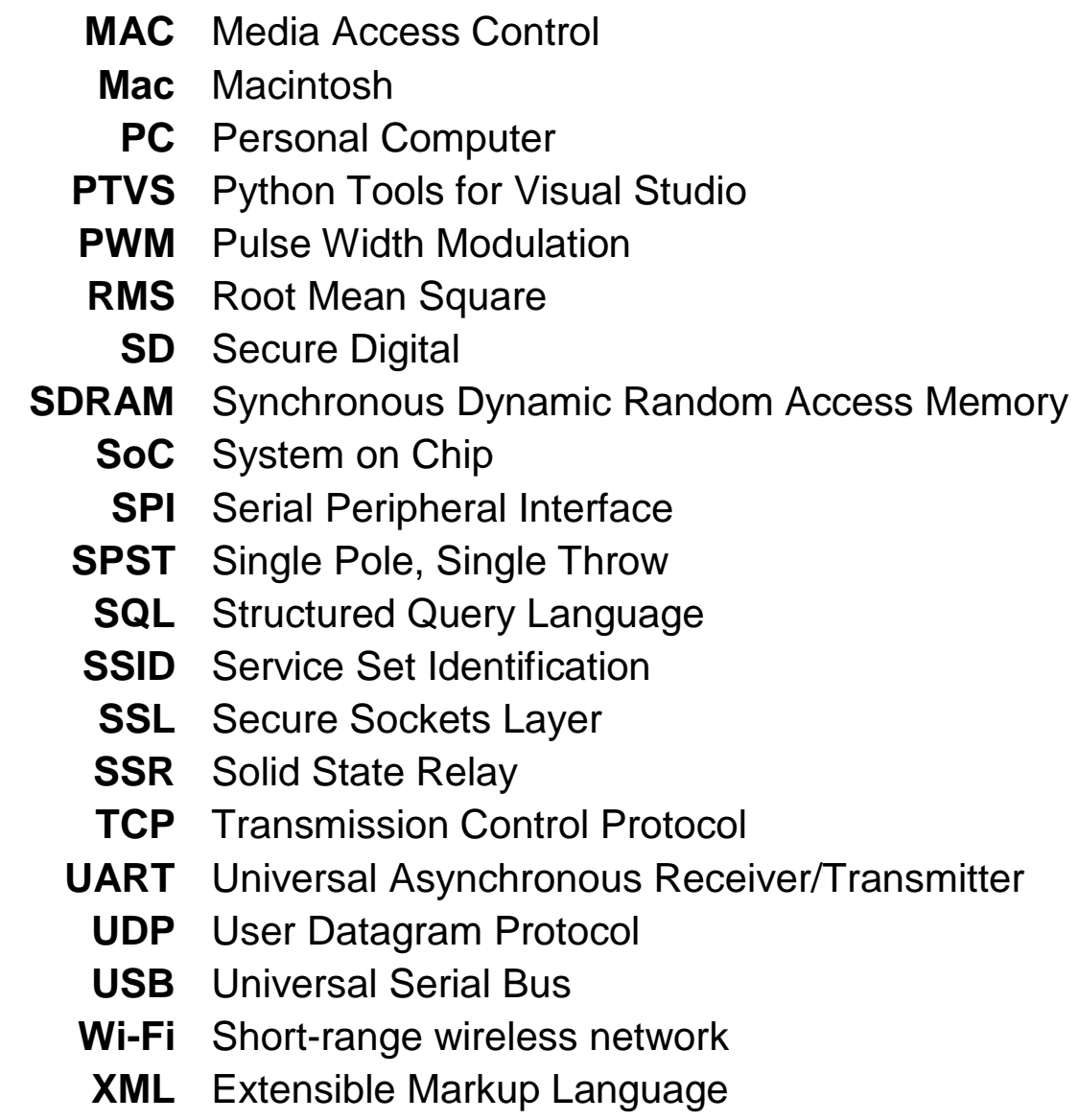

Glossary

Client Any device capable of displaying a webpage (laptop, phone, tablet, computer) that connects to the server in order to allow users to interact with the system.

Device Any mains electrical appliance (light, heater, radio, laptop, etc.) which is monitored and or controlled by a node.

Node The bespoke hardware developed for this project to monitor and switch devices. Nodes contain the logic, switching and monitoring circuitry. Nodes connect to the servervia Wi-Fi.

Server The entity (mainly software) that coordinates all of the functionality of the system. Nodes and clients connect to the server.

System The concept of one or more clients connecting to a single server which in turn controls one or more nodes each switching and monitoring one or more devices.

\section{References}

[1] Variability of wind power and other renewables: Management options and strategies The international Energy Agency, 2005, http://www.uwig.org/iea_report_on_variability.pdf. 
[2] Bass RJ, Malalasekera W, Willmot P. The impact of variable demand upon the performance of a combined cycle gas turbine (CCGT) power plant, Energy 2011; 36(4):1956-59.

[3] The Smart Grid: an introduction. US Department of Energy publication, 2009, http://energy.gov/oe/downloads/smart-grid-introduction-0.

[4] Lund H, Andersen AN, Østergaard PA, Mathiesen BV, Connolly D, From electricity smart grids to smart energy systems - a market operation based approach and understanding Energy 2012;42:96-102.

[5] Doostizadeh M, Ghasemi H, A day-ahead electricity pricing model based on smart metering and demand-side management. Energy 2012;46:221-230.

[6] Olmos L, Ruester S, Liong S, Glachant J, Energy efficiency actions related to the rollout of smart meters for small consumers, application to the Austrian system. Energy 2011;36:4396-4409.

[7] Pereira R, Figueiredo J, Melicio R, Mendes VMF, Martins J, Quadrado JC, Consumer energy management system with integration of smart meters. Energy Reports 2015;1:22-29.

[8] Beckel C, Sadamori L, Staake T, Santin, S, 2014, Revealing household characteristics from smart meter data. Energy 2014;78:397-410.

[9] Depuru SSSR, Wang L, Devabhaktuni V, Smart meters for power grid challenges issues advantages and status. Renewable and Sustainable Energy Reviews 2011;15(6):2736-2742.

[10] Vega AM, Santamaria F, Rivas E, Modeling for home electric energy management: A review. Renewable and Sustainable Energy Reviews 2015;52:948-959.

[11] Vassileva I, Wallin, F, Dahlquist E, Understanding energy consumption behaviour for future demand response strategy development. Energy 2012; 6(1):94-100.

[12] Zhao L, Zhang Ji-li, Liang R, Development of an energy monitoring system for large public buildings. Energy and Buildings 2013;66:41-48.

[13] Blumsack S, Fernandez A. Ready or not, here comes the smart grid!. Energy 2012; 37: 61-68.

[14] Alagoz BB, Kaygusuz A, Karabiber A, A user-mode distributed energy management architecture for smart grid applications. Energy 2012; 44:167-177

[15] Wissner M, The smart grid - a saucerful of secrets ?. Appl Energy 2011; 88: 25092518. 
[16] Batista NC, Melício R, Matias JCO, Catalão JPS, Photovoltaic and wind energy systems monitoring and building/home energy management using ZigBee devices within a smart grid. Energy 2013; 49: 306-315,

[17] Kim H, Lee SK, Kim H, Kim H, Implementing home energy management system with UPnP and mobile applications. Computer Communications 2012; 36 (1):51-62.

[18] Han D-M, Lim J-H, Smart Home Energy Management System using IEEE 802.15.4 and ZigBee. IEEE Transactions on Consumer Electronics 2010;10:1403-1409.

[19] Jang WS, Healy WM, Wireless sensor network performance metrics for building applications. Energy and Buildings 2009; 42(6):862-868.

[20] Han J, Choi C-S, Park W-K, Kim S-H, PLC-based photovoltaic system management for smart home energy management system, IEEE Transactions on Consumer Electronics 2014; 60(2):184-189.

[21] Papagiannis, GK, Papadopoulos, TA, Dovas, CD, Tsiamitros, DA. and Dokopoulos, PS, A PLC based energy consumption management system. Power line performance analysis: Field tests and simulation results, IEEE Power Technology Conference, 2005 Russia.

[22] Al-Mulla A., ElSherbini A., Demand management through centralized control system using power line communication for existing buildings, Energy Conversion and Management 2014; 79: 477-486.

[23] Yigit M, Gungor VC, Tuna G, Rangoussi M, Fadel E, Power line communication technologies for smart grid applications: A review of advances and challenges. Computer Networks 2014; Volume 70(9):366-383.

[24] Gold, S. Not-so-smart-meters, Network Security, 2009; 6:9-11.

[25] Mayers, RJ, Williams ED, Matthews HS. Scoping the potential of monitoring and control technologies to reduce energy use in homes, Energy and Buildings, 2010; 42: 563-569.

[26] Press WH, Teukolsky, SA, Vetterling WT, Flannery, B.P., Numerical Recipes: The Art of Scientific Computing, Second Edition, Cambridge University Press, 1992, Chapter 12.

[27] openPicus. (2013, 06 10). SoftAp Flyport WIFI G. Retrieved 07, 2013, from openPicus Wiki: http://wiki.openpicus.com/index.php/SoftAp Flyport WIFI G.

[28] SHARP. (2004). S102S01 Series, S202S01 Series. Datasheet, SHARP Corporation.

[29] Microchip. (2010). PIC24FJ256GA110 Family Data Sheet. 
[30] LEM - HX 03-P/SP2 - Current Transducer. Retrieved from http://www.lem.com/hq/en/component/option,com catalog/task,displaymodel/id,64.7 9.06.002.0/ also available at Onecall http://onecall.farnell.com/lem/hx-03-psp2/current-transducer/dp/1617419? Ntt=1617419.

[31] Texas Instruments. (July 2010). LM158, LM158A, LM258, LM258A, LM358, LM358A, LM2904, LM2904V, Dual operational amplifiers. Dallas, Texas 75265.

[32] Raspberry Pi - A credit Card Size Computer, http://www.raspberrypi.org/, accessed Feb 2015.

[33] Broadcom. (2012). BCM2835 ARM Peripherals. Broadcom Europe Ltd. 406 Science Park Milton Road Cambridge CB4 OWW.

[34] Wheezy Raspbian http://www.raspbian.org/RaspbianAbout, accessed Feb 2015.

[35] Prodigit Electronics Co.LTD. (2009). Model 2000MU Plug-In Power Monitor. Datasheet.

[36] Microsoft, Python Tools for Visual Studio. Retrieved 23-07-2013, from CodePlex: http://pytools.codeplex.com/.

[37] Python, pyton.org, MySQL-python, accessed Feb 2013, https://pypi.python.org/pypi/MySQL-python

[38] NumPy.org, accessed Feb. 2013, http://www.numpy.org/.

[39] SciPi.org, accessed Feb. 2013, http://scipy.org/

[40] Samba, Samba.org., Feb. 2013, https://www.samba.org/samba/

[41] CSS Tutorial, W3Schools.com, http://www.w3schools.com/css/

[42] Notepad++, https://notepad-plus-plus.org/.

[43] Highcharts.com, accessed Feb. 2013, http://www.highcharts.com/

[44] Department of Energy \& Climate Change. (December 2012). Quarterly Energy Prices. https://www.gov.uk/government/statistics/quarterly-energy-prices-december2012.

[45] Department of Energy and Climate Change (DECC). (2011). Guidelines to Defra / DECC's GHG Conversion Factors for Company Reporting, https://www.gov.uk/government/uploads/system/uploads/attachment data/file/6931 4/pb13625-emission-factor-methodology-paper-110905.pdf.

[46] Eric Gamess, Rina Surós. (2008, November). An upper bound model for TCP and UDP throughput in IPv4 and IPv6. Journal of Network and Computer Applications, 31(4), 585-602. 


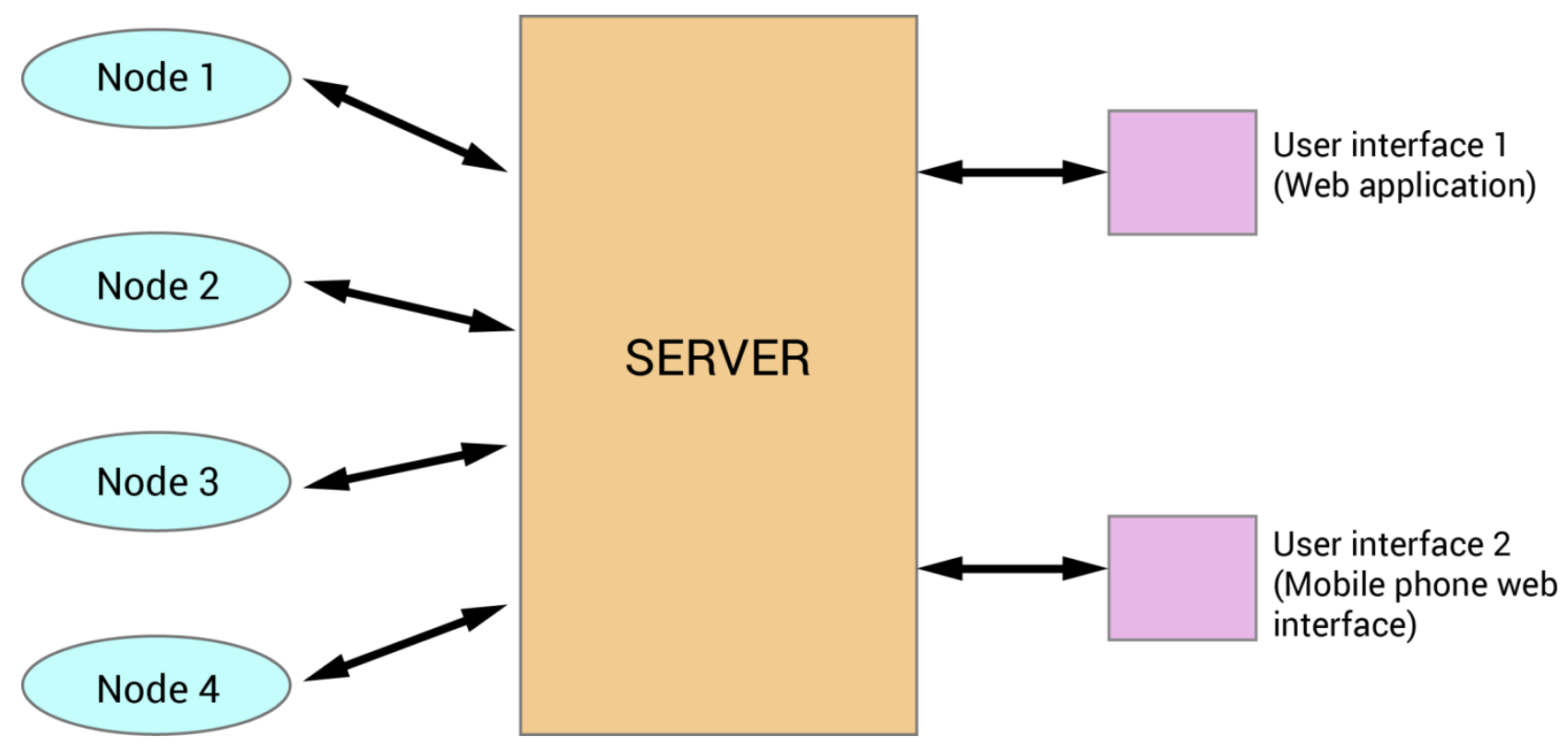

Figure 1. An illustration of the overall system concept

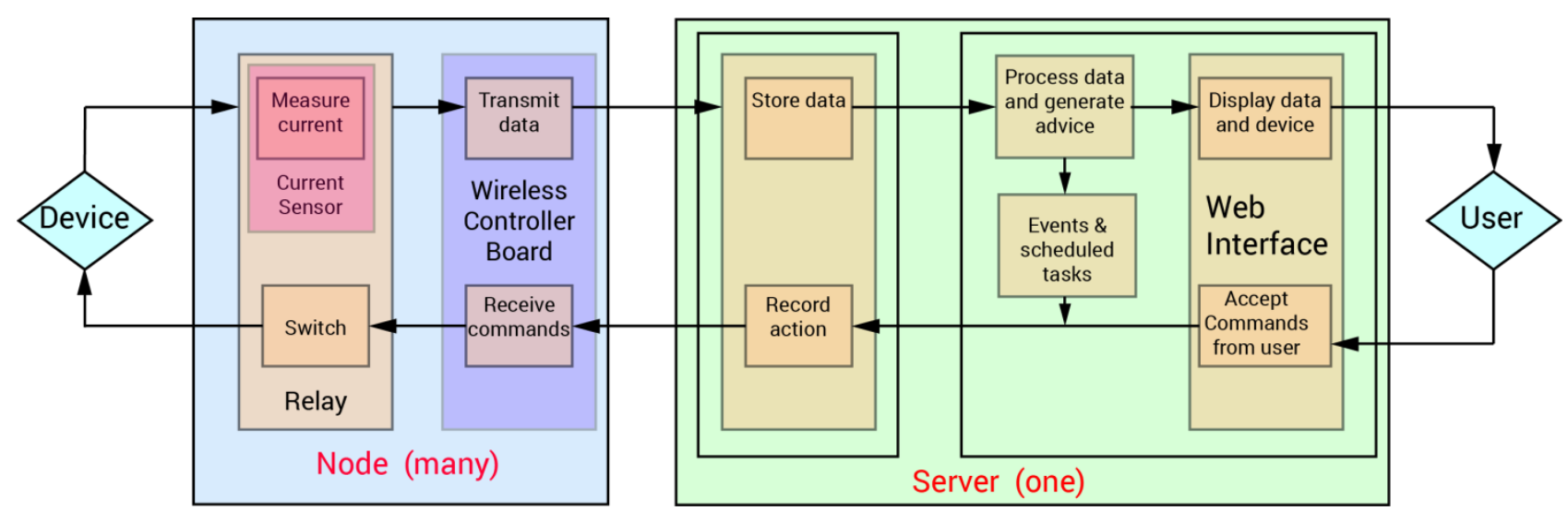

Figure 2 Detailed system layout 


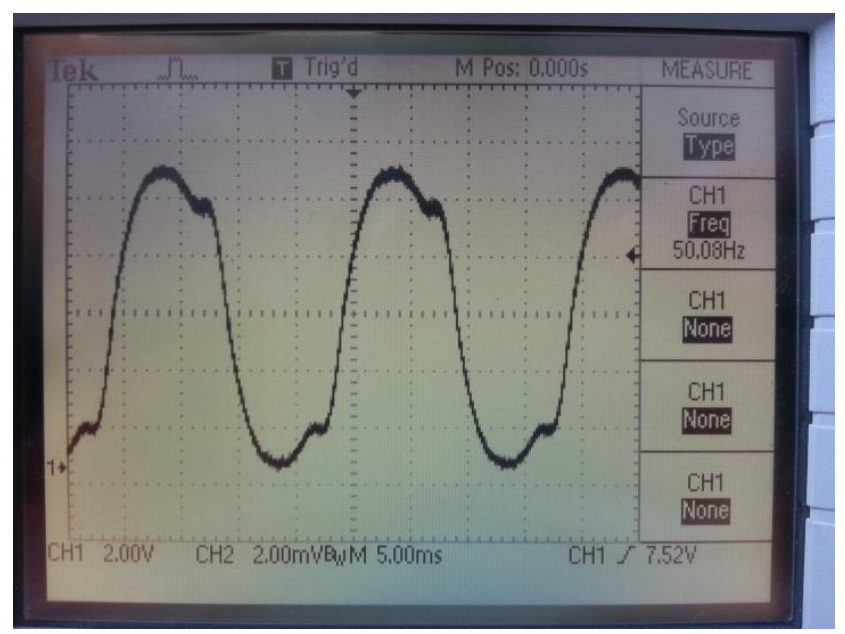

Overhead Projector (Fan and Light)

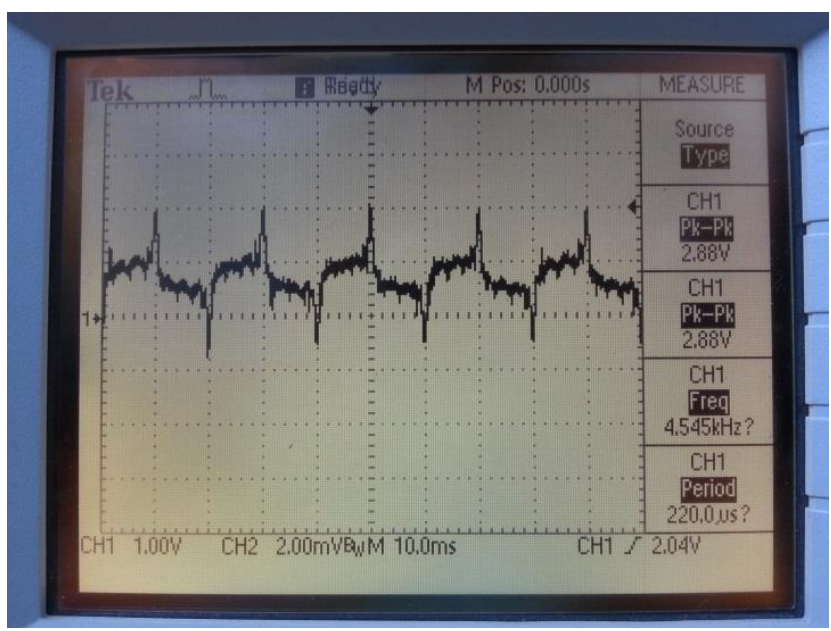

AV Equipment

Figure 3. Measured current transducer waveform for two devices

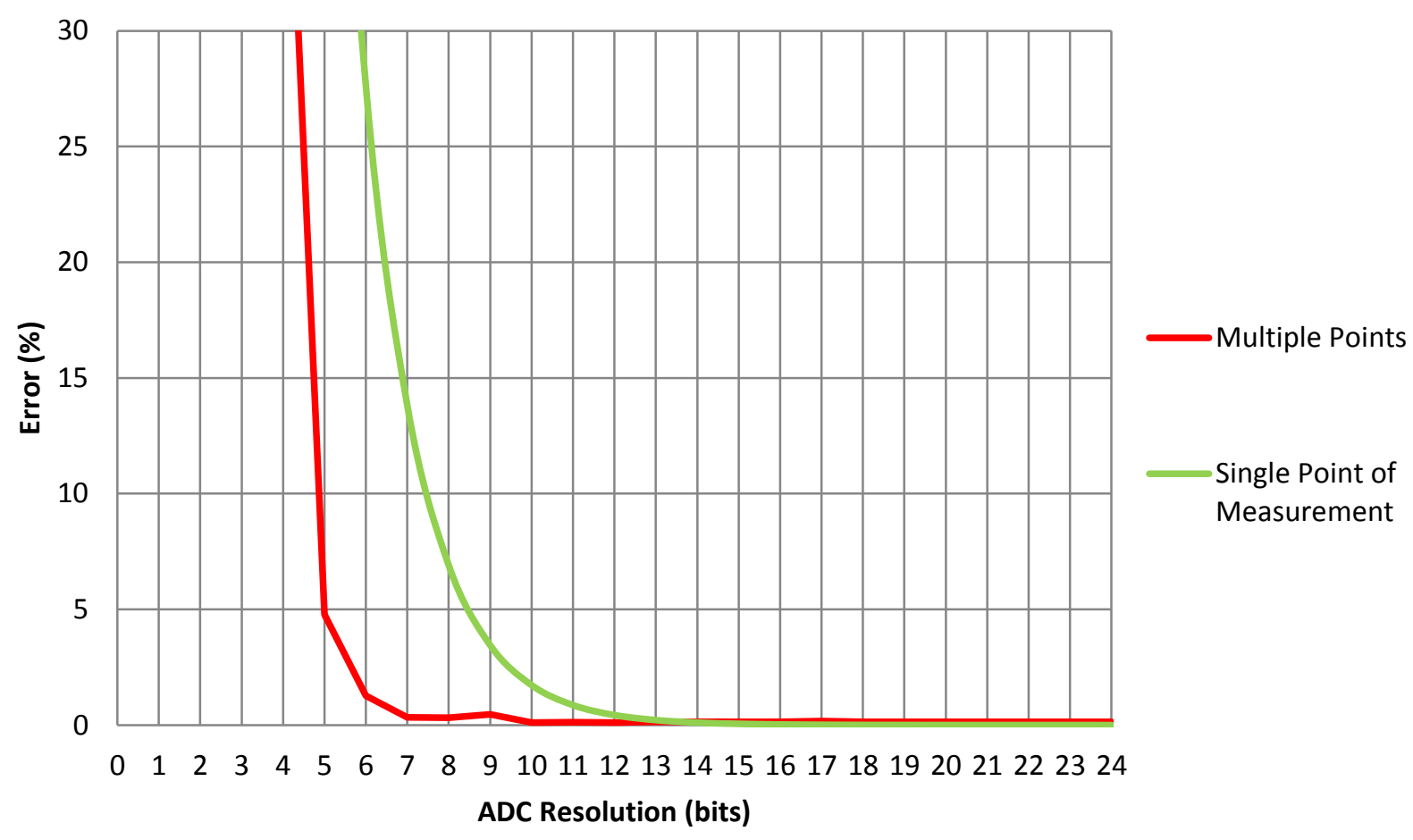

Figure 4 - Error as a function of ADC resolution 


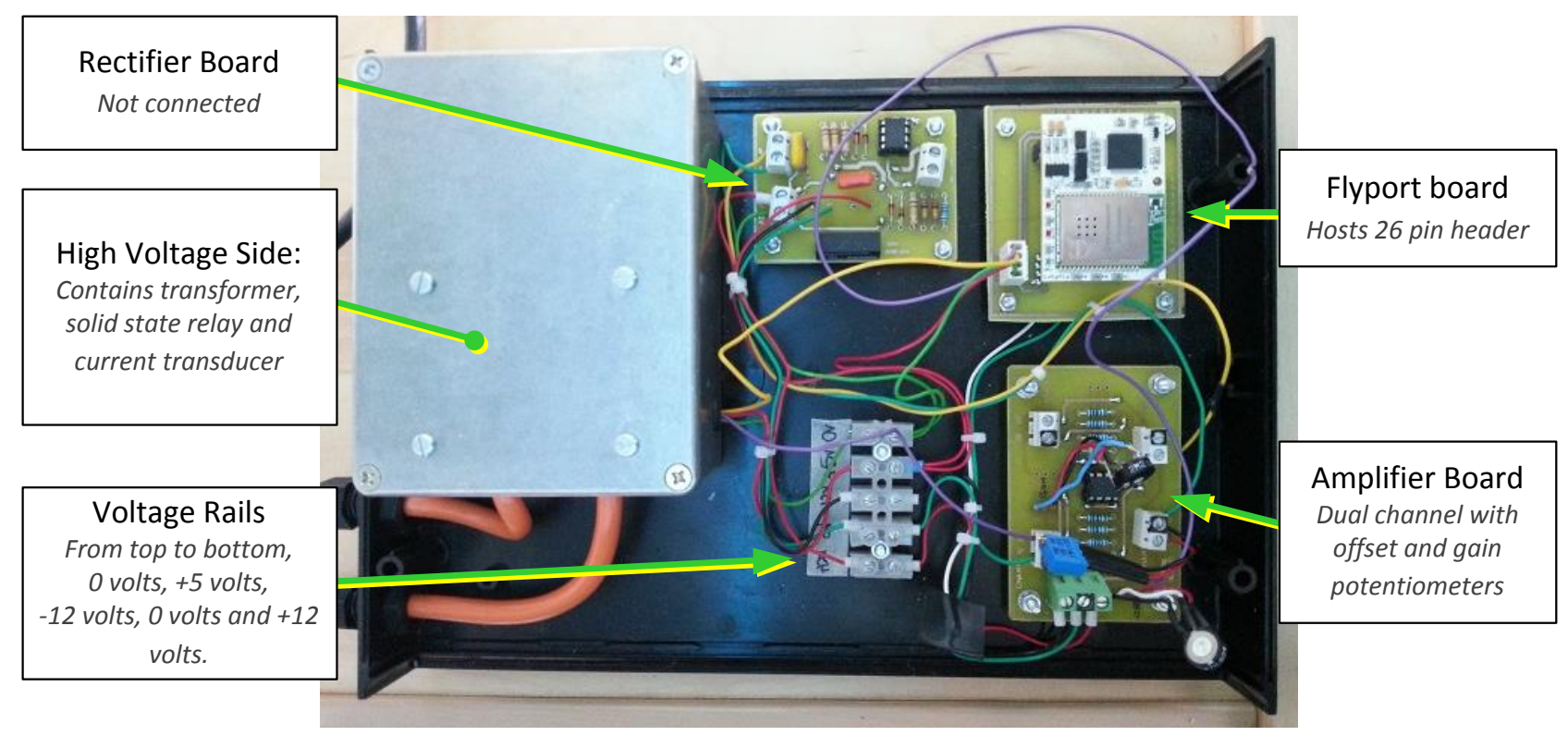

Figure 5 Final prototype with cover removed 


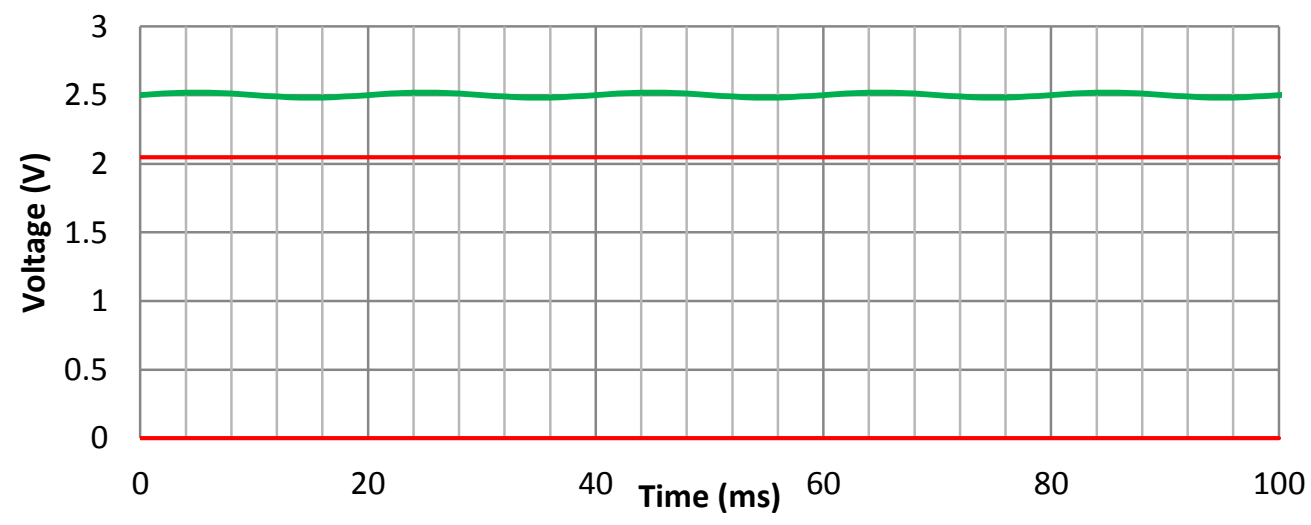

Figure 6 - Current transducer output

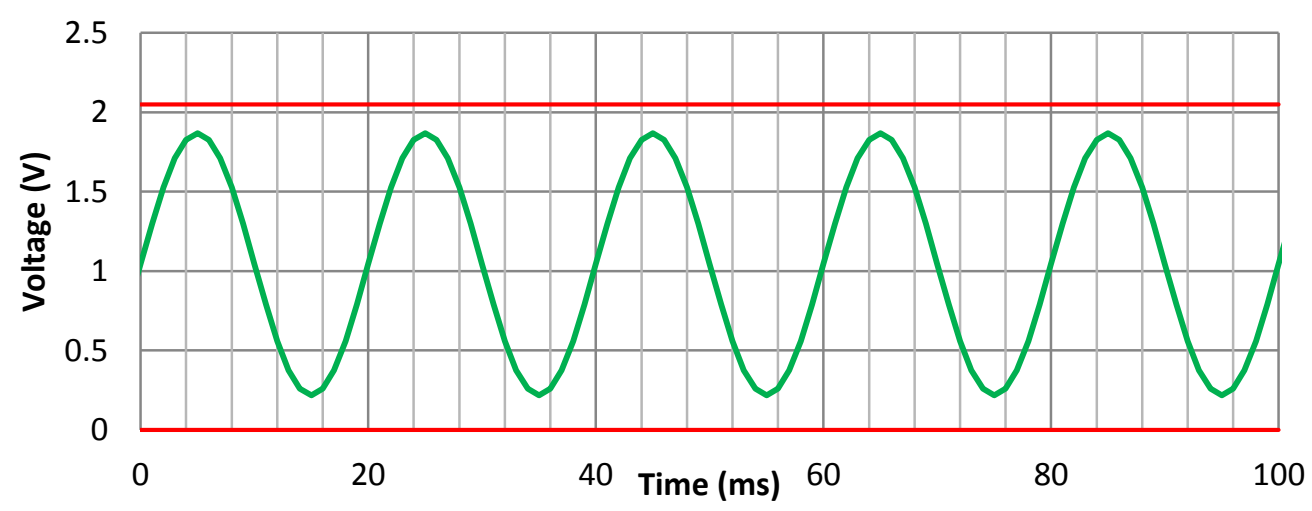

Figure 7 - Amplifier output (to Flyport) 


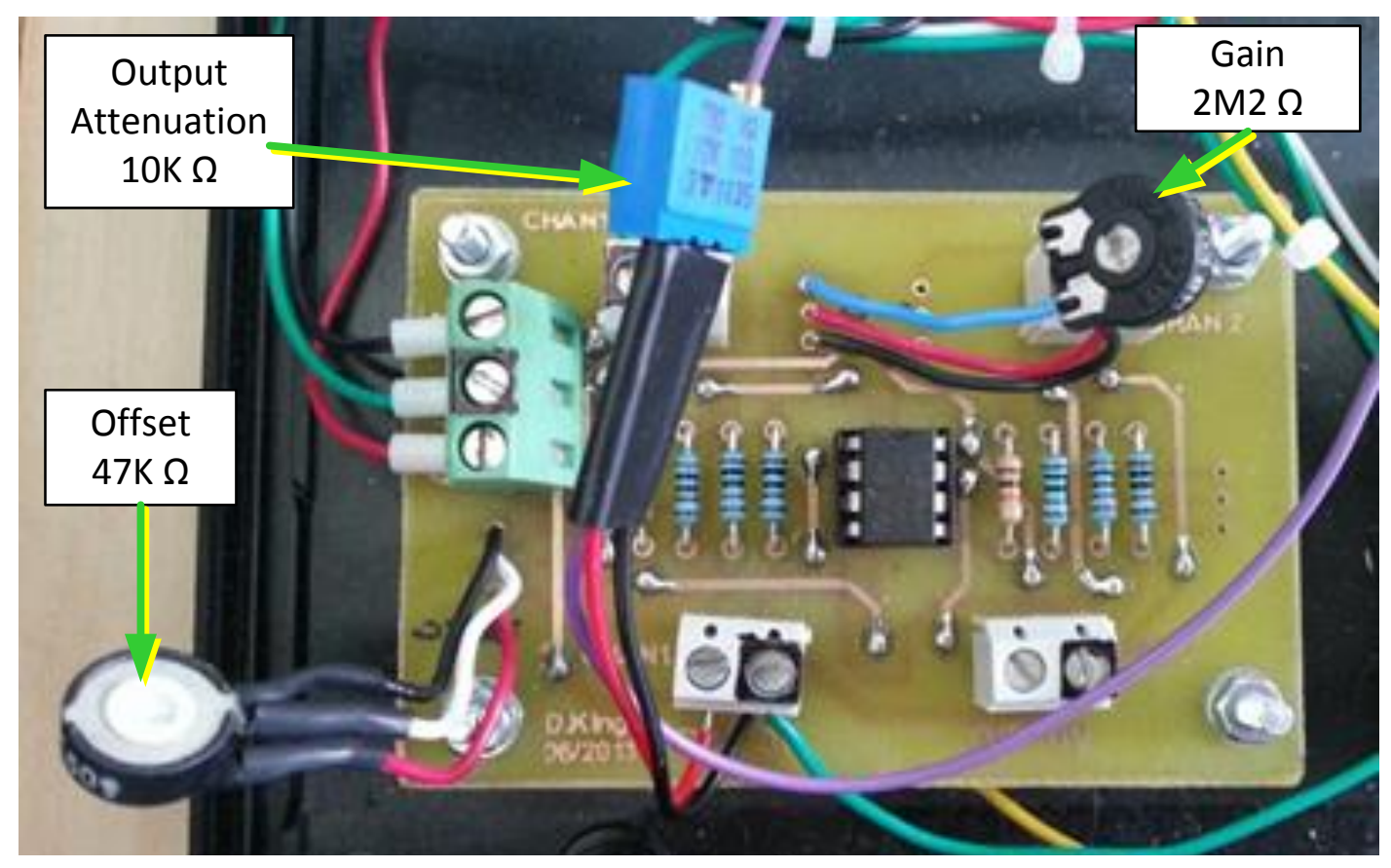

Figure 8 Node amplifier board showing important potentiometers

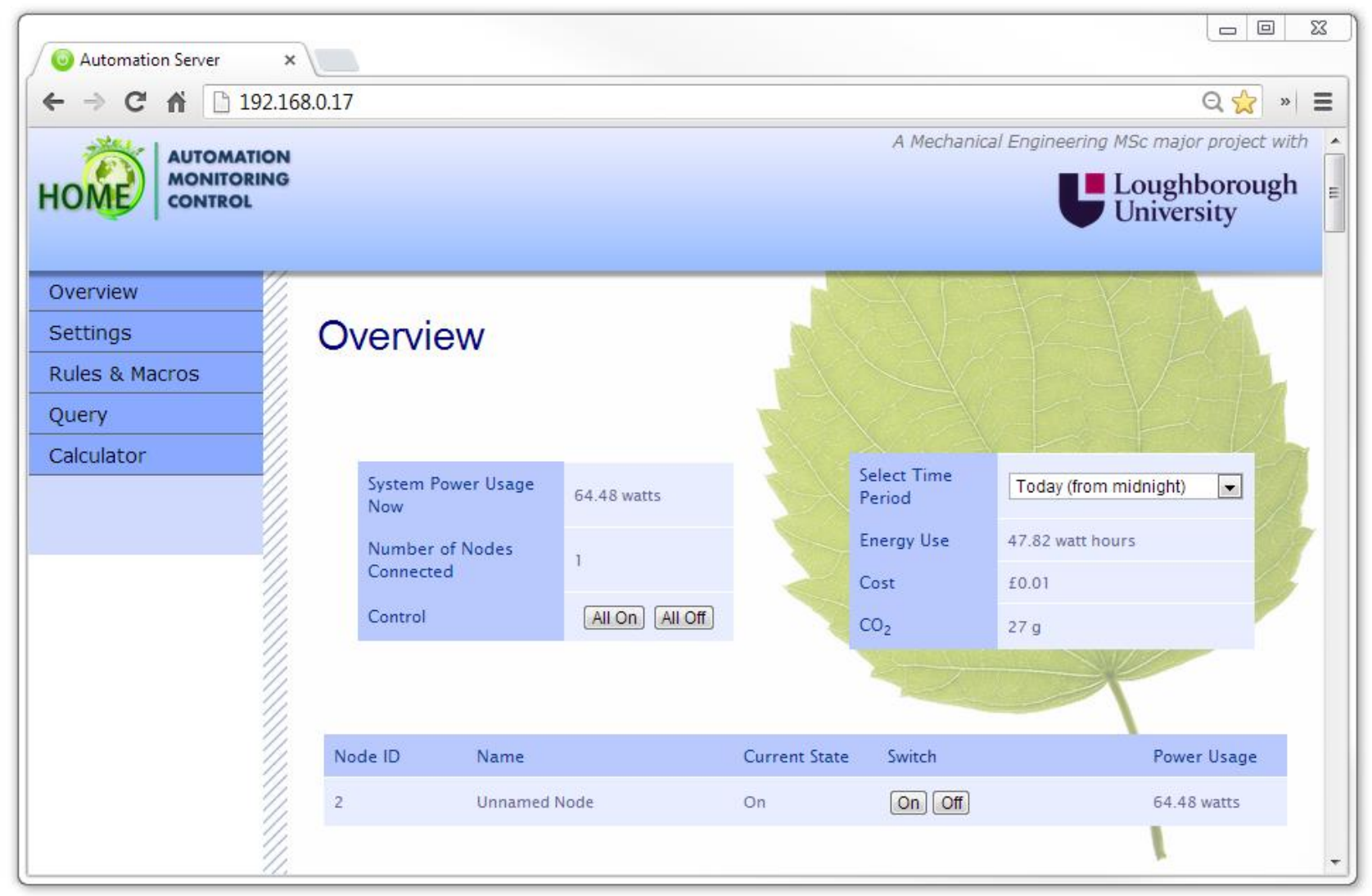

Figure 9 - Overview page 


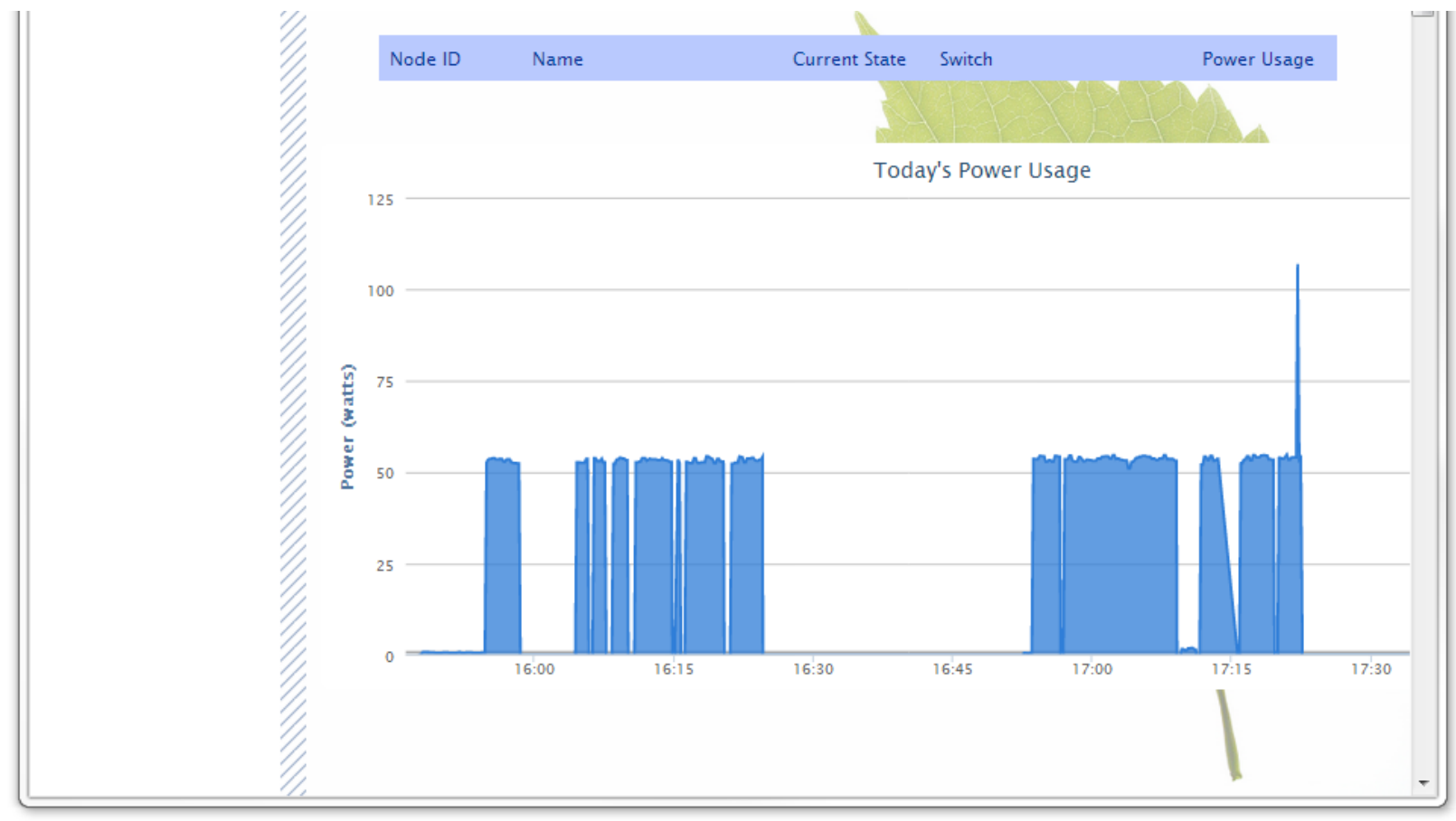

Figure 10 - Power usage graph

\begin{tabular}{|c|c|c|}
\hline \multirow{2}{*}{ Select Time Period } & Today (from midnight) & - \\
\hline & \multicolumn{2}{|l|}{ Today (from midnight) } \\
\hline Energy Use & \multicolumn{2}{|l|}{ This Week (from Monday) } \\
\hline Cost & \multicolumn{2}{|l|}{$\begin{array}{l}\text { Previous week } \\
\text { Previous month }\end{array}$} \\
\hline $\mathrm{CO}_{2}$ & \\
\hline & $\begin{array}{l}\text { Last } 7 \text { days } \\
\text { Last } 30 \text { days } \\
\text { This Year } \\
\text { All }\end{array}$ & \\
\hline Switch & Power Usage & \\
\hline
\end{tabular}

Figure 11 - Time period menu 


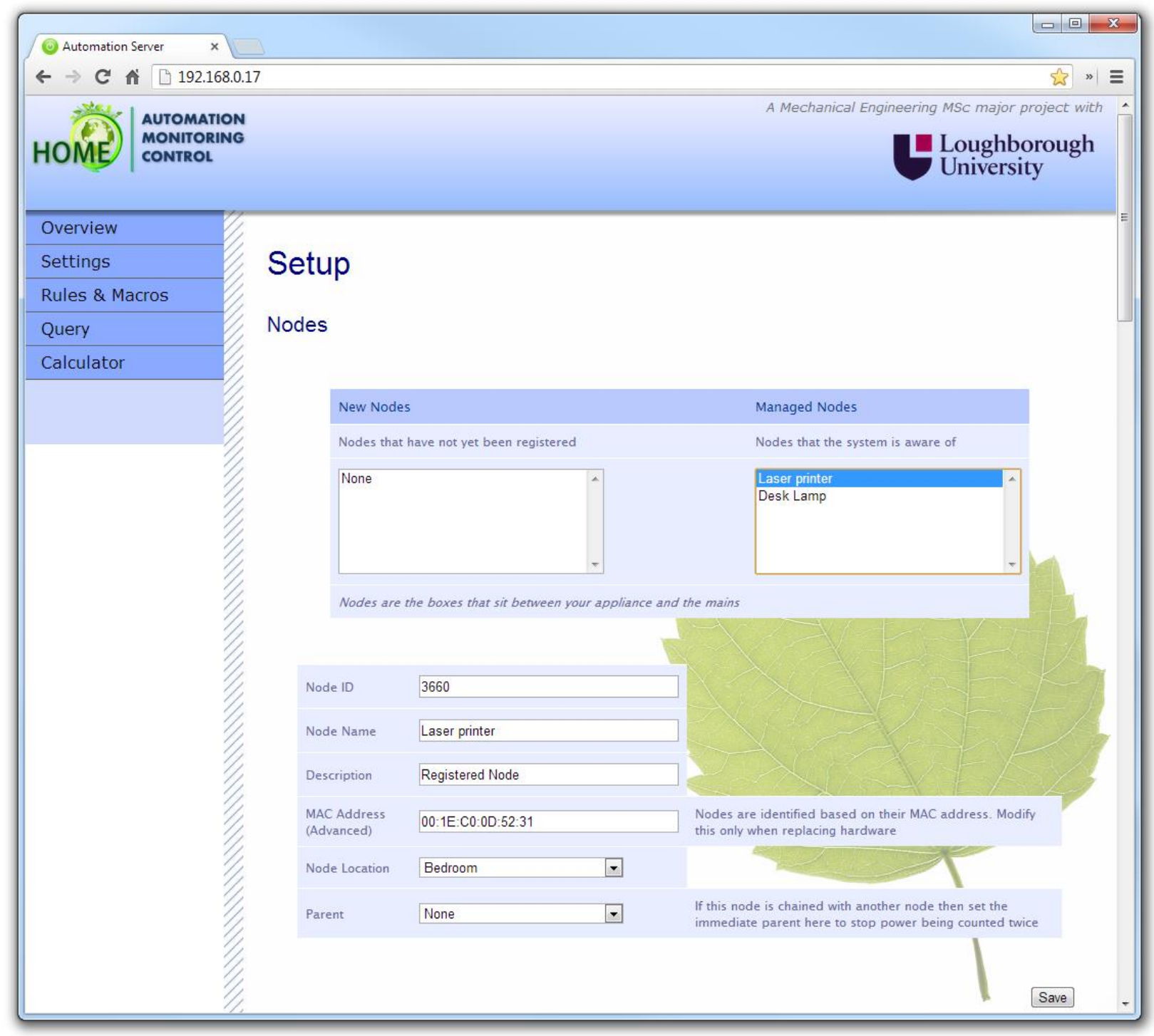

Figure 12 - Settings page 


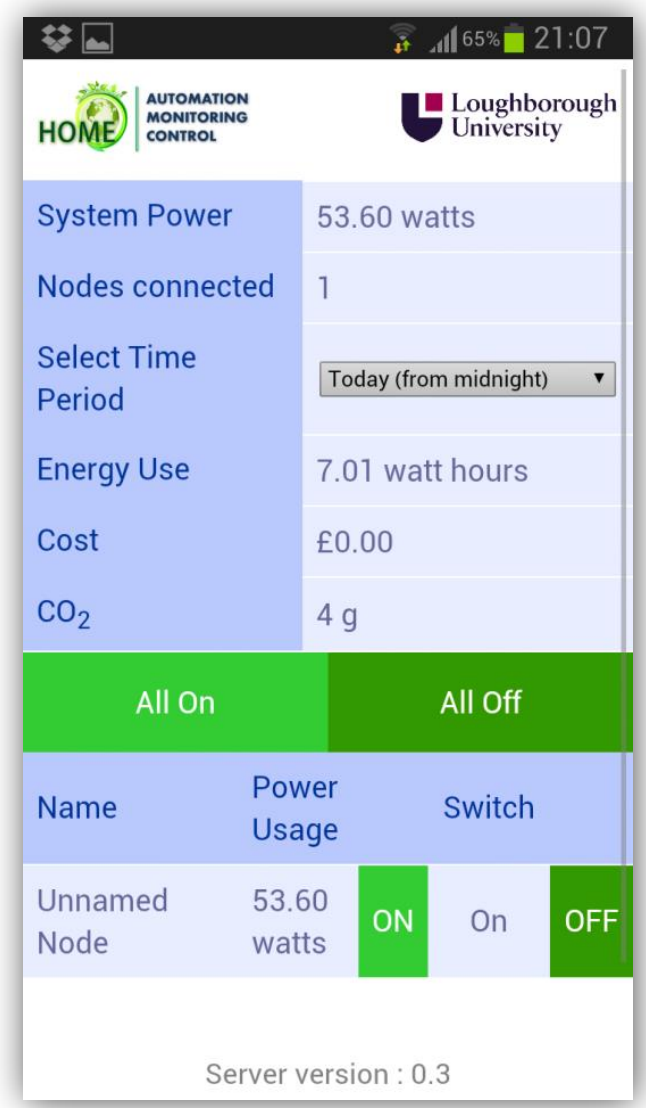

Figure 13 - Mobile version of the overview page

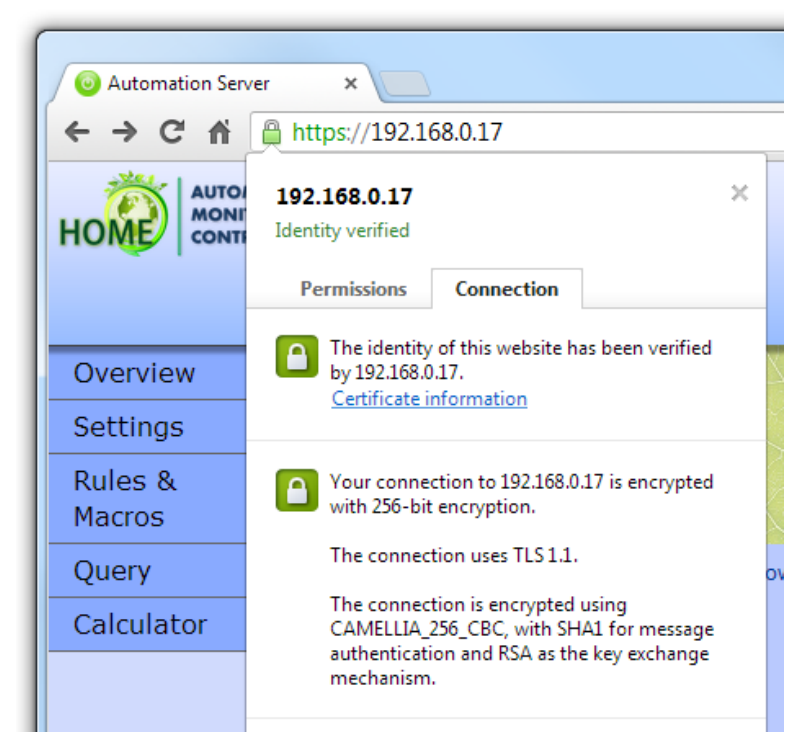

Figure 14 - HTTPS connection information in Google Chrome 


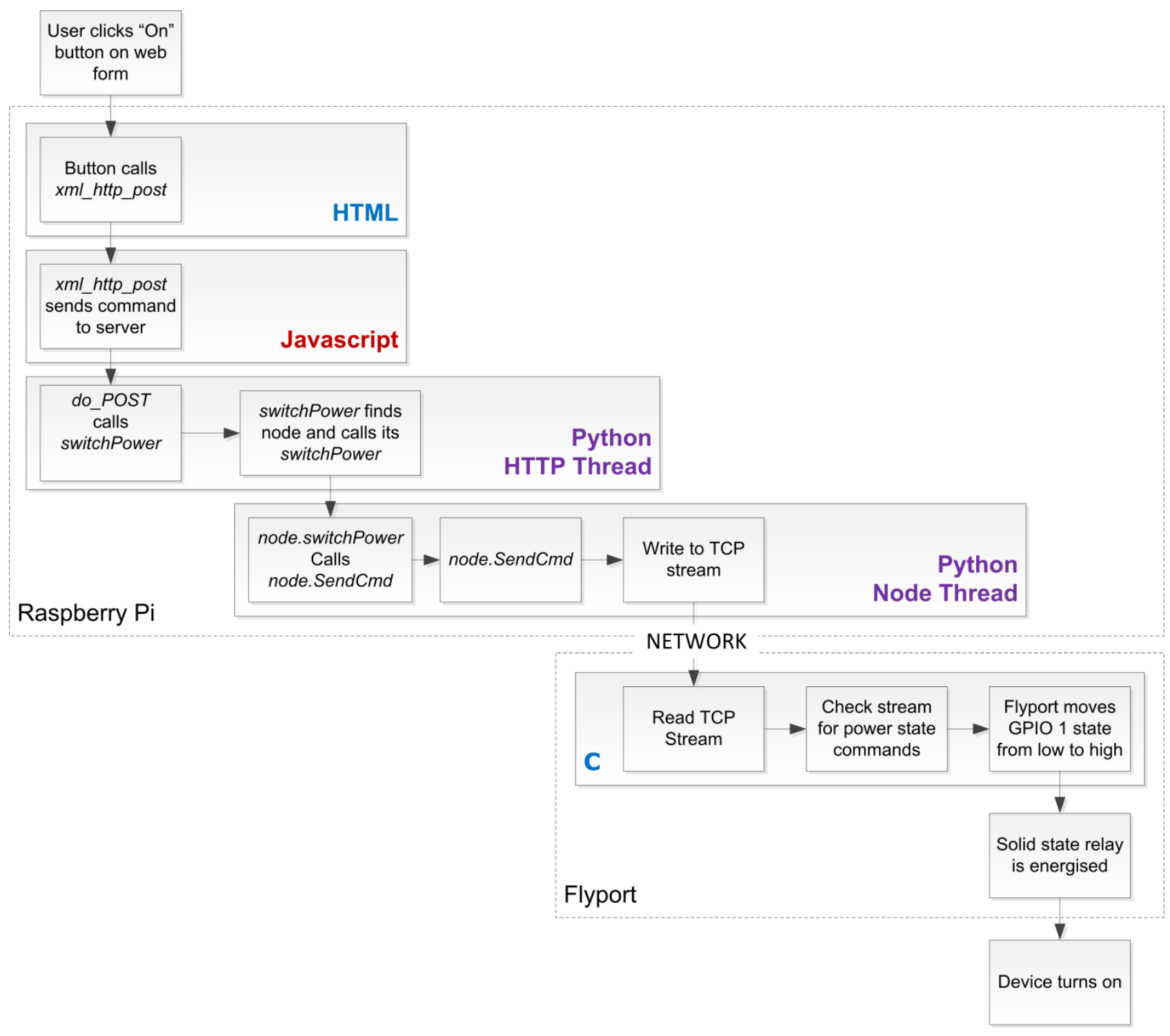

Figure 15 - Power switch input / output path 


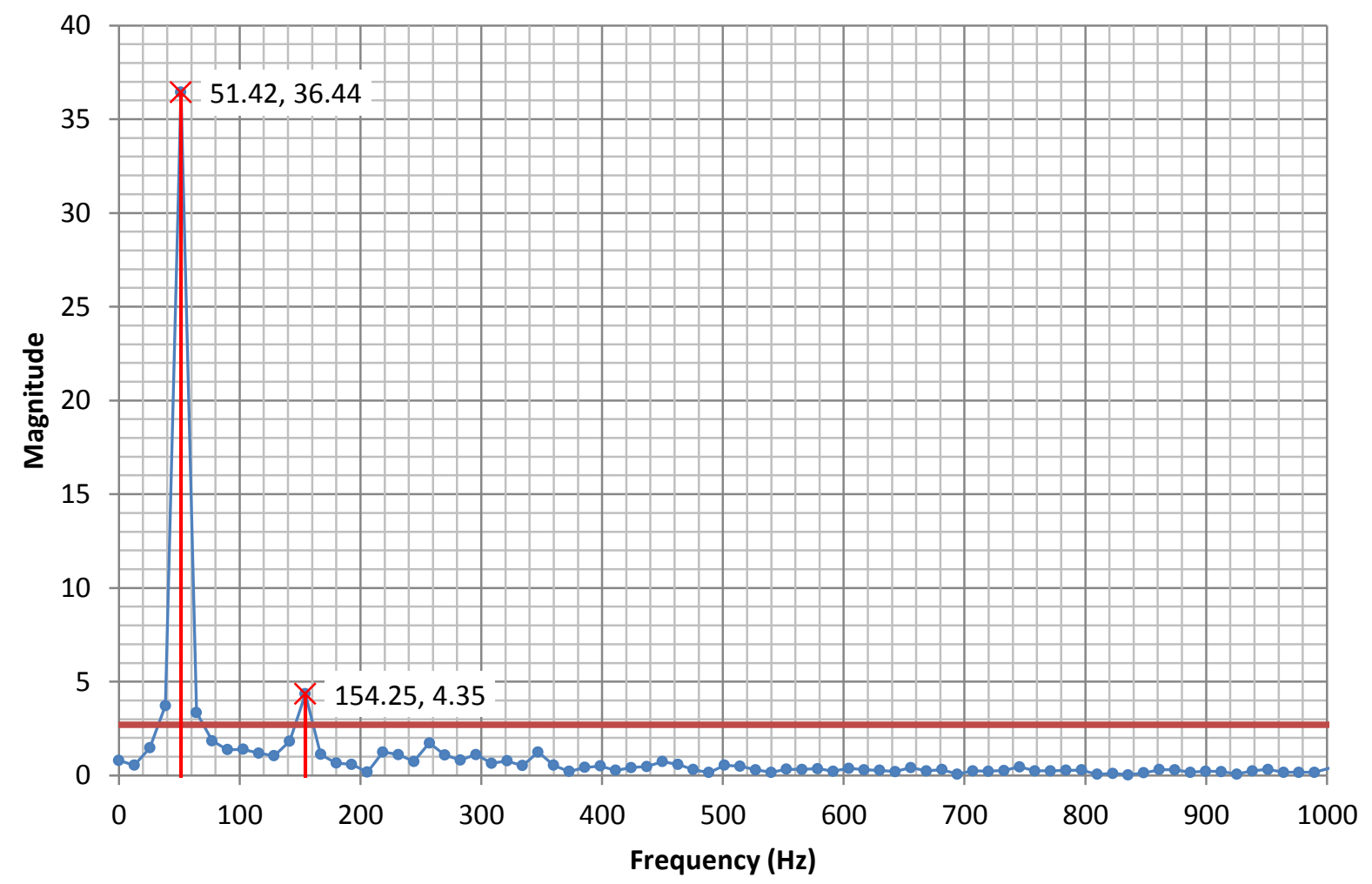

Figure 16 - Frequency domain of test signal

\section{Devices}

Lights, Fans, AV Equiptment etc

This system can try to identify connected devices based on the way they use power.

(Device identification only works with devices that draw a static load.)

\begin{tabular}{|c|c|c|}
\hline Device is currently connected to Node & Unnamed Node & 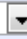 \\
\hline Device Name & Low Energy Light & \\
\hline \multicolumn{3}{|c|}{$\begin{array}{l}\text { Please plug the device into the node, ensure it is switched on (node will turn } \\
\text { on automatically) and press Start }\end{array}$} \\
\hline \multirow[t]{2}{*}{ Progress } & 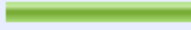 & \\
\hline & $\begin{array}{l}49.98 \mathrm{~Hz} @ 8.37 \\
159.92 \mathrm{~Hz} @ 4.32\end{array}$ & \\
\hline Frequencies & & \\
\hline
\end{tabular}

These are the major

Figure 17 - Device profiling tool 


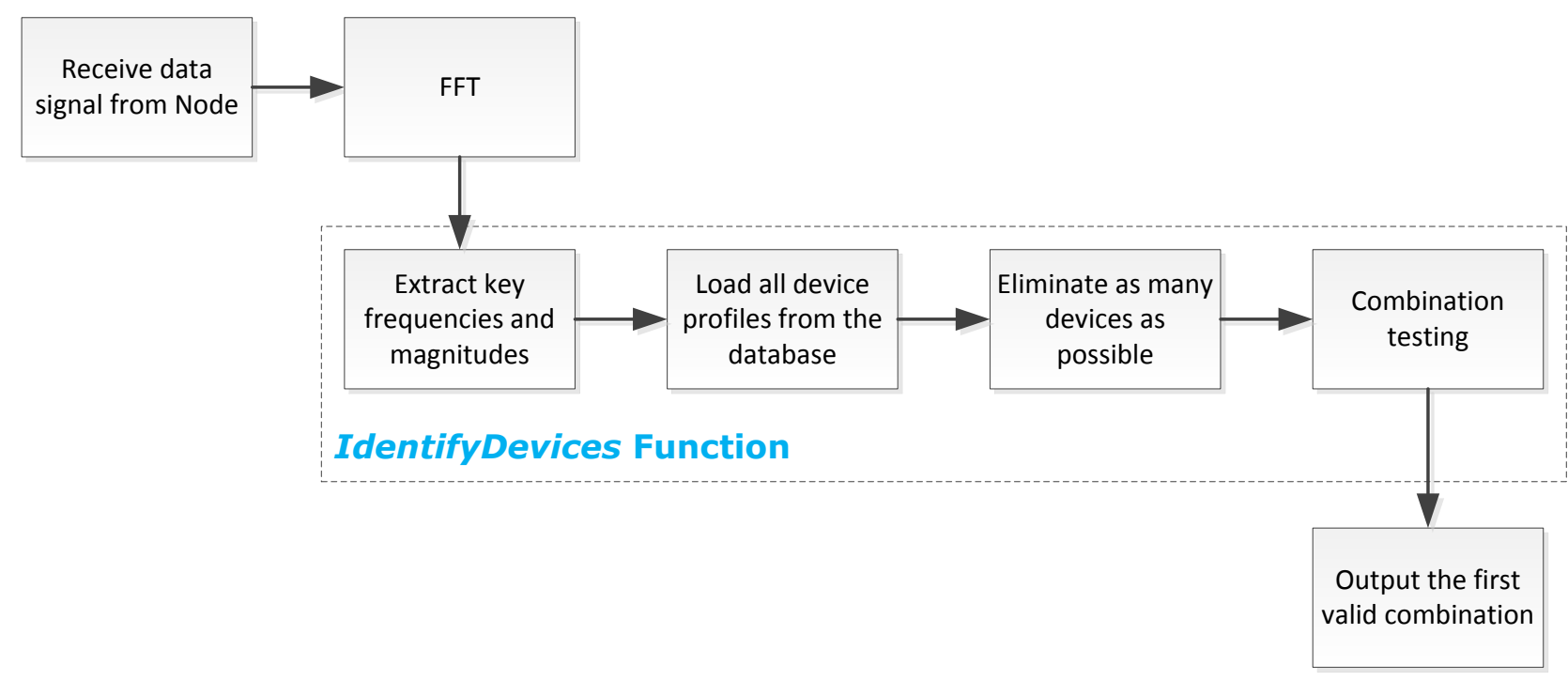

Figure 18 - Device recognition procedure

\section{Test Devices}

Use this tool to see what is currently connected to the system

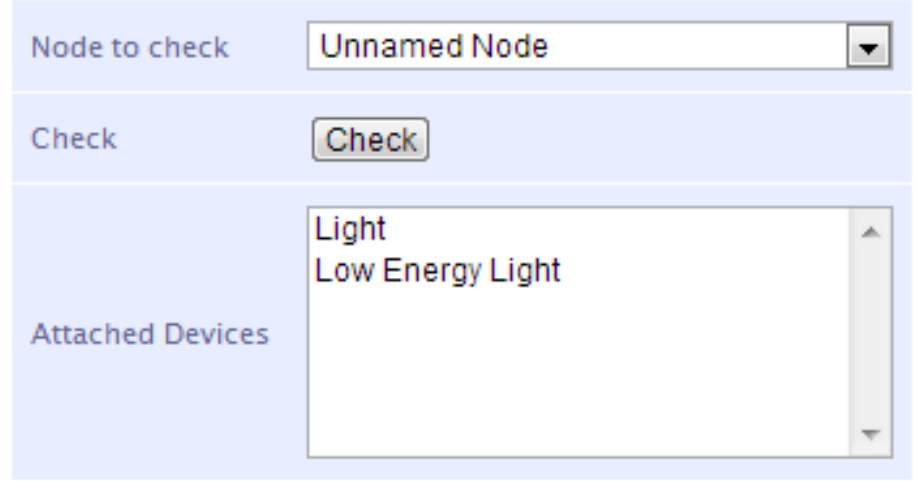

Figure 19 - Device recognition test tool 


\begin{tabular}{|l|ll|l|}
\hline $\begin{array}{l}\text { Potential Device } \\
1\end{array}$ & Potential Device & $\begin{array}{l}\text { Bit encoded } \\
\text { string (binary) }\end{array}$ & $\begin{array}{l}\text { Integer Value of bit } \\
\text { encoded string }\end{array}$ \\
\hline Off & Off & 00 & 0 \\
\hline Off & On & 01 & 1 \\
\hline On & Off & 10 & 2 \\
\hline On & On & 11 & 3 \\
\hline
\end{tabular}

Table 1 - Device states 